\title{
SOME HILBERT SPACES OF ENTIRE FUNCTIONS. III
}

BY

\author{
LOUIS DE BRANGES( $\left.{ }^{1}\right)$
}

We continue the study of Hilbert spaces, whose elements are entire functions, and which have these three properties:

(H1) Whenever $F(z)$ is in the Hilbert space and has a nonreal zero $w$, the function $F(z)(z-\bar{w}) /(z-w)$ is in the Hilbert space and has the same norm as $F(z)$.

(H2) For each nonreal number $w$, the linear functional defined on the Hilbert space by $F(z) \rightarrow F(w)$ is continuous.

(H3) Whenever $F(z)$ is in the Hilbert space, the function $F^{*}(z)=\bar{F}(\bar{z})$ is in the Hilbert space and has the same norm as $F(z)$.

If $E(z)$ is an entire function such that

$$
|E(\bar{z})|<|E(z)|
$$

for $y>0(z=x+i y)$, we write

$$
E(z)=A(z)-i B(z)
$$

where $A(z)$ and $B(z)$ are entire functions which are real for real $z$, and

$$
K(w, z)=[B(z) \bar{A}(w)-A(z) \bar{B}(w)] /[\pi(z-\bar{w})] .
$$

Let $\mathfrak{F C}(E)$ be the Hilbert space of entire functions $F(z)$ such that

$$
\|F\|^{2}=\int|F(t)|^{2}|E(t)|^{-2} d t<\infty
$$

with integration on the real axis, and

$$
|F(z)|^{2} \leqq\|F\|^{2} K(z, z)
$$

for all complex $z$. For each complex number $w, K(w, z)$ belongs to $\mathfrak{H C}(E)$ as a function of $z$ and

$$
F(w)=\langle F(t), K(w, t)\rangle
$$

for every $F(z)$ in $\mathcal{H C}(E)$. It was shown in [7] that every nonzero Hilbert space of entire functions which satisfies $(\mathrm{H} 1),(\mathrm{H} 2)$, and $(\mathrm{H} 3)$ is equal isometrically to some such $\mathfrak{H C}(E)$.

The typical example of such a space occurs in Fourier analysis, and then there is a family $(E(a, z))$ of entire functions satisfying (1), $a>0$ :

Received by the editors January 5, 1961.

( ${ }^{1}$ ) This research was supported by the United States Air Force under contract No. AF49(638)-253. 


$$
\begin{gathered}
E(a, z)=\exp (-i a z) \\
A(a, z)=\cos (a z), \quad B(a, z)=\sin (a z) .
\end{gathered}
$$

When $a \leqq b, \mathcal{H}(E(a))$ is contained isometrically in $\mathcal{H}(E(b))$, and every one of these spaces is contained isometrically in $L^{2}(-\infty, \infty)$. A necessary and sufficient condition that an entire function $F(z)$ belong to $\mathcal{F}(E(a))$ is that it be of the form

$$
\pi F(z)=\int f(t) \cos (t z) d t+\int g(t) \sin (t z) d t,
$$

where $f(t)$ and $g(t)$ are measurable complex valued functions of $t \geqq 0$, which are square integrable and which vanish a.e. outside of $[0, a]$; in this case,

$$
\pi \int|F(t)|^{2} d t=\int|f(t)|^{2} d t+\int|g(t)|^{2} d t .
$$

This is a relatively trivial interpretation of the celebrated Theorem $\mathrm{X}$ of Paley and Wiener [12]. There is a closely analogous integral representation valid in the most general space $\mathfrak{F}(E)$. For simplicity of presentation, we will suppose that $E(z)$ has no real zeros and that $E(0)=1$.

Let

$$
m(t)=\left(\begin{array}{ll}
\alpha(t) & \beta(t) \\
\beta(t) & \gamma(t)
\end{array}\right)
$$

be a matrix valued function of $t>0$, where $\alpha(t), \beta(t), \gamma(t)$ are real valued, absolutely continuous functions of $t>0$ such that

$$
\alpha^{\prime}(t) \geqq 0, \quad \gamma^{\prime}(t) \geqq 0, \quad \beta^{\prime}(t)^{2} \leqq \alpha^{\prime}(t) \gamma^{\prime}(t)
$$

a.e. for $t>0$,

$$
\alpha(t)>0 \text { for } t>0 \text { and } \lim _{t \searrow 0} \alpha(t)=0
$$

and

$$
\lim _{t \rightarrow \infty}[\alpha(t)+\gamma(t)]=\infty
$$

A real number $b>0$ is said to be singular with respect to $m(t)$ if it belongs to an open interval $(a, c)$ in which $\alpha^{\prime}(t), \beta^{\prime}(t), \gamma^{\prime}(t)$ are equal a.e. to constant multiples of a single function and

$$
\beta^{\prime}(t)^{2}=\alpha^{\prime}(t) \gamma^{\prime}(t)
$$

a.e. Otherwise, a number $b>0$ is said to be regular with respect to $m(t)$. Let $L^{2}(m)$ be the Hilbert space of (equivalence classes of) pairs $(f(t), g(t))$ of measurable complex valued functions of $t \geqq 0$ such that 


$$
\|(f, g)\|^{2}=\int(f(t), g(t)) d m(t)(f(t), g(t))^{-}<\infty
$$

Here,

$$
(f(t), g(t))^{-}=\left(\begin{array}{l}
\bar{f}(t) \\
\bar{g}(t)
\end{array}\right)
$$

and the integral is to be interpreted as

$$
\int(f(t), g(t)) m^{\prime}(t)(f(t), g(t))-d t
$$

By the equivalence of $\left(f_{1}(t), g_{1}(t)\right)$ and $\left(f_{2}(t), g_{2}(t)\right)$ in an interval $(a, b)$, we mean that

$$
\int_{a}^{b}(f(t), g(t)) d m(t)(f(t), g(t))^{-}=0
$$

where

$$
(f(t), g(t))=\left(f_{2}(t), g_{2}(t)\right)-\left(f_{1}(t), g_{1}(t)\right) .
$$

Let $L_{0}^{2}(m)$ be the closed subspace of $L^{2}(m)$ formed by the elements $(f, g)$ of $L^{2}(m)$ such that $(f(t), g(t))$ is equivalent to a constant in any interval $(a, b)$ containing only singular points with respect to $m(t)$. Since $\beta(t)$ is real valued by hypothesis, $(\bar{f}(t), \bar{g}(t))$ belongs to $L^{2}(m)$ whenever $(f(t), g(t))$ belongs to $L^{2}(m)$ and these elements have the same norm; if one belongs to $L_{0}^{2}(m)$, so does the other. Let $H$ be the transformation in $L_{0}^{2}(m)$ defined by $\left(f_{2}, g_{2}\right)$ $=H\left(f_{1}, g_{1}\right)$ if $\left(f_{1}, g_{1}\right)$ and $\left(f_{2}, g_{2}\right)$ are elements of $L_{0}^{2}(m)$, and $\left(f_{1}, g_{1}\right)$ is (equivalent to a pair of functions) such that $f_{1}(t)$ is continuous for $t>0, g_{1}(t)$ is continuous for $t \geqq 0, g_{1}(0)=0$, and

$$
\left(f_{1}(b), g_{1}(b)\right) I-\left(f_{1}(a), g_{1}(a)\right) I=\int_{a}^{b}\left(f_{2}(t), g_{2}(t)\right) d m(t)
$$

whenever $0<a<b<\infty$. Here,

$$
I=\left(\begin{array}{rr}
0 & -1 \\
1 & 0
\end{array}\right)
$$

and (5) is to be taken in the sense that

$$
\begin{aligned}
& g_{1}(b)-g_{1}(a)=\int_{a}^{b}\left[f_{2}(t) \alpha^{\prime}(t)+g_{2}(t) \beta^{\prime}(t)\right] d t, \\
& f_{1}(a)-f_{1}(b)=\int_{a}^{b}\left[f_{2}(t) \beta^{\prime}(t)+g_{2}(t) \gamma^{\prime}(t)\right] d t .
\end{aligned}
$$


Both integrals are absolutely convergent by the Schwarz inequality, because of (2) and the finiteness of $\left\|\left(f_{2}, g_{2}\right)\right\|$. The transformation $H$ is well-defined because the step functions which belong to $L^{2}(m)$ are dense in $L^{2}(m)$. Note that if $\left(f_{1}, g_{1}\right)$ is in the domain of $H$, then so is $\left(\bar{f}_{1}, \bar{g}_{1}\right)$; if $\left(f_{2}, g_{2}\right)=H\left(f_{1}, g_{1}\right)$, then $\left(\bar{f}_{2}, \bar{g}_{2}\right)=H\left(\bar{f}_{1}, \bar{g}_{1}\right)$.

By a Hilbert space, we mean a complete inner product space over the complex numbers, with no restriction on dimension. The spaces which actually occur are separable, but they may have finite dimension. Our transformations are linear, but they need not be everywhere defined or bounded.

Our generalization of the Paley-Wiener theorem will take place in several stages, as announced in [9]. In the first theorem, we develop the concept of a one-parameter family of Hilbert spaces of entire functions in analogy with the family $E(a, z)=\exp (-i a z)$. In the second theorem, we show that such a family is present whenever we are given a single Hilbert space of entire functions and a measure which determines its norm. The third theorem is the proper generalization of the Fourier transform in the Paley-Wiener fashion. It also describes the significance of this transformation for the underlying self-adjoint transformation $H$. In this formulation, we are equally indebted to Paley and Wiener [12] and to Stone [13].

THEOREM I. Let $m(t)$ be a matrix valued function of $t>0$ which satisfies (2), (3), and (4). Suppose that there exists a family $(E(t, z))$ of entire functions satisfying (1) with no real zeros, such that $E(t, 0)=1, t>0$, such that for each complex number $w, E(t, w)$ is a continuous function of $t>0$,

$$
(A(b, w), B(b, w)) I-(A(a, w), B(a, w)) I=w \int_{a}^{b}(A(t, w), B(t, w)) d m(t)
$$

whenever $0<a<b<\infty$, and

$$
\lim _{a \searrow 0} K(a, w, w)=0 .
$$

Then, when $a<b$ are regular points with respect to $m(t), \mathcal{H}(E(a))$ is contained isometrically in $\operatorname{HC}(E(b))$. For all nonreal numbers $w$,

$$
\lim _{b \rightarrow \infty} K(b, w, w)=\infty .
$$

There is a unique non-negative measure $\mu$ on the Borel sets of the real line such that

$$
\int\left(1+t^{2}\right)^{-1}|E(a, t)|^{2} d \mu(t)<\infty
$$

for each regular $a>0$, and

$$
\frac{y}{\pi} \int \frac{|E(a, t)|^{2} d \mu(t)}{(t-x)^{2}+y^{2}}=\lim _{b \rightarrow \infty} \frac{y}{\pi} \int \frac{|E(a, t)|^{2}|E(b, t)|^{-2}}{(t-x)^{2}+y^{2}} d t
$$


for $y>0$. When $a>0$ is regular with respect to $m(t), \operatorname{HC}(E(a))$ is contained isometrically in $L^{2}(\mu)$. The union of the spaces $\mathfrak{H C}(E(a))$, with a regular, is dense in $L^{2}(\mu)$.

THEOREM II. Let $E(z)$ be an entire function which satisfies (1) and has no real zeros, such that $E(0)=1$. Let $\nu$ be a non-negative measure on the Borel sets of the real line such that $\mathfrak{H C}(E)$ is contained isometrically in $L^{2}(\nu)$. Then, $E(z)$ $=E(c, z)$ and $\nu=\mu$ for some choice of $m(t)$ and $E(t, z)$ as in Theorem I, and some $c>0$ which is regular with respect to $m(t)$.

Let $\chi(a, t)$ be the function which is equal to 1 when $t \leqq a$ and equal to 0 when $t>a$.

TheOREM III. Let $m(t)$ and $E(t, z)$ be as in Theorem I.

(A) Let $c>0$ be regular with respect to $m(t)$. Then,

$$
\chi(c, t)(A(t, w), B(t, w))
$$

belongs to $L_{0}^{2}(m)$ as a function of $t$ for every complex number $w$. For each element $(f, g)$ of $L_{0}^{2}(m)$ which is equivalent to zero outside of $[0, c]$, define a corresponding "eigentransform" $F(z)$ by

$$
\pi F(w)=\int(f(t), g(t)) d m(t)(A(t, \bar{w}), B(t, \bar{w}))^{-}
$$

for all complex w. Then, $F(z)$ is an entire function, it belongs to $\operatorname{HC}(E(c))$, and

$$
\pi \int|F(t)|^{2} d \mu(t)=\int(f(t), g(t)) d m(t)(f(t), g(t))^{-} .
$$

If $G(z)$ is in $\mathcal{H C}(E(c))$, then $G(z)$ is equal to $F(z)$ for some such choice of $(f, g)$. If $F(z)$ is the eigentransform of $(f, g)$, then $F^{*}(z)$ is the eigentransform of $(\bar{f}, \bar{g})$.

(B) Let $c>0$ be regular with respect to $m(t)$. Let $\left(f_{1}, g_{1}\right)$ be an element of $L_{0}^{2}(m)$ which is equivalent to zero outside of $[0, c]$, and let $F_{1}(z)$ be the corresponding eigentransform. A necessary and sufficient condition that $\left(f_{1}, g_{1}\right)$ be in the domain of $H$ and that $\left(f_{1}(c), g_{1}(c)\right)=0$ is that $z F_{1}(z)$ belong to $\operatorname{HC}(E(c))$. In this case, let $\left(f_{2}, g_{2}\right)=H\left(f_{1}, g_{1}\right)$ and let $F_{2}(z)$ be the corresponding eigentransform. Then, $F_{2}(z)=z F_{1}(z)$ for all complex $z$.

(C) If $(f, g)$ is in $L_{0}^{2}(m)$, the corresponding eigentransform $F(x)$, defined by

$$
F(x)=\lim _{c \rightarrow \infty} \int_{0}^{c}(f(t), g(t)) d m(t)(A(t, x), B(t, x))^{-},
$$

exists with convergence in the metric of $L^{2}(\mu)$, and (10) holds. Every element $G(x)$ of $L^{2}(\mu)$ is equal, a.e. with respect to $\mu$, to the eigentransform $F(x)$ of an element $(f, g)$ of $L_{0}^{2}(m)$. If $F(x)$ is the eigentransform of $(f, g)$, then $\bar{F}(x)$ is the eigentransform of $(\bar{f}, \bar{g})$.

(D) Let $\left(f_{1}, g_{1}\right)$ be an element of $L_{0}^{2}(m)$ and let $F_{1}(x)$ be the corresponding 
eigentransform. A necessary and sufficient condition that $\left(f_{1}, g_{1}\right)$ be in the domain of $H$ is that $x F_{1}(x)$ be in $L^{2}(\mu)$. In this case, let $\left(f_{2}, g_{2}\right)=H\left(f_{1}, g_{1}\right)$ and let $F_{2}(x)$ be the corresponding eigentransform. Then, $F_{2}(x)=x F_{1}(x)$ a.e. with respect to $\mu$.

Probably the most interesting of these three theorems is Theorem II. From a single Hilbert space of entire functions we are able to extrapolate a whole family of such Hilbert spaces with isometric inclusions. In other words, the choice of a single function $E(z)$ satisfying (1) invokes a giant apparatus similar to that of Fourier analysis. For the spaces containing $\mathfrak{H C}(E)$, we use a measure which determines the norm of $\mathfrak{H C}(E)$. All such measures were previously determined in Theorem $\mathrm{V}$ of [8]. No choice of measure is needed to obtain the spaces contained in $\mathfrak{H C}(E)$. This half of the theorem has previously been obtained in Theorem $\mathrm{V}$ of $[10]$ in the case that $E(z)$ has genus 0 or 1 . The proofs do not provide any effective method for making the construction. If an entire function $E(z)$ satisfying (1) is given, say by a Hadamard factorization, we have no effective general method of finding an associated oneparameter family of entire functions satisfying (1). Nevertheless, such calculations can be made in special cases. Since these computations are quite complicated, we have preferred to restrict ourselves to a single typical example, which we give in great detail, rather than discuss a large number of cases less completely.

The gamma function $\Gamma(z)$ may be used to construct an interesting example of a Hilbert space of entire functions. From our point of view, it is more natural to work with its reciprocal $S(z)$, which is an entire function. Since the gamma function satisfies the identity

$$
\Gamma(z+1)=z \Gamma(z)
$$

the reciprocal satisfies the identity

$$
S(z)=z S(z+1) .
$$

It follows that $S(z)$ has zeros at the nonpositive integers, and we are led to conjecture a factorization of the form

$$
S(z)=e^{\gamma 0 z} z \prod_{1}^{\infty}(1+z / n) e^{-z / n},
$$

which is known to hold for a suitable choice of the real number $\gamma_{0}$, Euler's constant. See Whittaker and Watson [15, Chapter XII]. On taking the logarithm and the derivative of each side, we find that

$$
\operatorname{Re} i S^{\prime}(z) / S(z)=y|z|^{-1}+\sum_{1}^{\infty} y|z+n|^{-2}>0
$$

for $y>0$. It follows that the function $S^{\prime}(z)-i S(z)$ satisfies (1). It has no real 
zeros and it has value 1 at the origin. The construction follows the standard proof of Laguerre's theorem, as in, say, Boas [1, p. 23].

If $E(z)=S^{\prime}(z)-i S(z)$, the Hilbert space $\pi c(E)$ has the functions

$$
\pi^{-1 / 2} S(z) /(z+n) \quad(n=0,1,2, \cdots)
$$

as an orthonormal basis. The identity $z S(z+1)=S(z)$ implies that the space has these two properties.

(I) The function $z F(z+1)$ belongs to $\mathfrak{H C}(E)$ whenever $F(z)$ belongs to $\mathfrak{H C}(E)$ and it has the same norm as $F(z)$.

(II) Every element $G(z)$ of $\mathfrak{F}(E)$ such that $G(0)=0$ is of the form $G(z)$ $=z F(z+1)$ for some corresponding $F(z)$ in $\mathcal{H}(E)$. A study of these two properties leads to a generalization of the gamma function identity.

THEOREM IV. Let $E(z)$ be an entire function which satisfies (1), such that $E(0)=1$. A necessary and sufficient condition that $\mathfrak{F C}(E)$ satisfy (I) and (II) is that there exist a matrix

$$
P=\left(\begin{array}{ll}
p & q \\
r & s
\end{array}\right)
$$

of real numbers, with $\operatorname{det} P=1$, such that

$$
z(A(z+1), B(z+1)) P=(A(z), B(z))\left(\begin{array}{cc}
1 & 0 \\
-z^{-1} \alpha^{-1} & 1
\end{array}\right)
$$

for all complex $z$, where $\alpha=B^{\prime}(0)>0$.

In the gamma function case, the identity (12) is satisfied with

$$
P=1, \quad A(z)=S^{\prime}(z), \quad B(z)=S(z), \quad \alpha=1 .
$$

It is then equivalent to the two scalar equations

$$
S(z)=z S(z+1), \quad S^{\prime}(z)-S(z) / z=z S^{\prime}(z+1) .
$$

As we have seen, the first equation is an expression of the gamma function identity. The second equation follows from the first on differentiating each side with respect to $z$. Although we choose to regard (12) as a generalization of the gamma function identity, it differs from the usual formulation of this identity in two respects. In the first place, it is an identity involving entire functions. In the second place, it is a vector identity involving a pair of entire functions. Nevertheless, because of the role of this identity in Theorem IV, it does have much of the flavor of the classical identity.

Since $S(z)$ is an entire function of genus $1, S^{\prime}(z)-i S(z)$ has genus 1 . We may apply Theorem $\mathrm{V}$ of $[10]$ to produce a family $(E(t, z))$ of entire functions satisfying (1), $0<t \leqq 1$, such that

$$
E(1, z)=S^{\prime}(z)-i S(z) .
$$


We will have a matrix valued function $m(t)$ defined for $0<t \leqq 1$, so as to satisfy (2), (3), (6), and

$$
\int_{0}^{1} \alpha(t) d \gamma(t)<\infty
$$

and

$$
\lim _{t>0} E(t, z) \exp [\beta(t) z]=1
$$

for all complex $z$. If $a$ is regular with respect to $m(t), \mathfrak{H}(E(a))$ is contained isometrically in $\mathfrak{H}(E(1))$. Since $\mathcal{H}(E(1))$ satisfies (I) and (II), it is tempting to conjecture that $\mathfrak{H C}(E(a))$ satisfies (I) and (II). If this is true, then by Theorem IV there is a matrix $P(a)$ of real numbers, with det $P(a)=1$, such that

$$
z(A(a, z+1), B(a, z+1)) P(a)=(A(a, z), B(a, z))\left(\begin{array}{cc}
1 & 0 \\
-z^{-1} \alpha(a)^{-1} & 1
\end{array}\right)
$$

If one differentiates each side formally with respect to $a$ and simplifies using (6) and (16), one is led after some calculation to conjecture the matrix identity

$$
\begin{array}{r}
-(z+1)\left(\begin{array}{cc}
1 & 0 \\
-z^{-1} \alpha(a)^{-1} & 1
\end{array}\right) P(a)^{-1} m^{\prime}(a) I P(a)+\left(\begin{array}{cc}
1 & 0 \\
-z^{-1} \alpha(a)^{-1} & 1
\end{array}\right) P(a)^{-1} P^{\prime}(a) \\
=-z m^{\prime}(a) I\left(\begin{array}{ccc}
1 & 0 \\
-z^{-1} \alpha(a)^{-1} & 1
\end{array}\right)+\left(\begin{array}{cc}
0 & 0 \\
\alpha^{\prime}(a) z^{-1} \alpha(a)^{-2} & 0
\end{array}\right),
\end{array}
$$

which turns out to be equivalent to the equation

$$
P(a)^{-1} P^{\prime}(a)=\left(\begin{array}{cc}
\beta^{\prime}(a) & -\alpha^{\prime}(a) \\
\gamma^{\prime}(a) & -\beta^{\prime}(a)
\end{array}\right)+\left(\begin{array}{cc}
-\alpha^{\prime}(a) \alpha(a)^{-1} & 0 \\
-2 \beta^{\prime}(a) \alpha(a)^{-1} & \alpha^{\prime}(a) \alpha(a)^{-1}
\end{array}\right)
$$

and the requirement that $P(a)$ commute with $m^{\prime}(a) I$. After some calculation, one finds a solution of the form

$$
P(a)=1+a \log a\left(\begin{array}{cc}
v(a) & -1 \\
v^{2}(a) & -v(a)
\end{array}\right)
$$

with

$$
\alpha(a)=a, \quad \beta^{\prime}(a)=v(a), \quad \gamma^{\prime}(a)=v^{2}(a)
$$

and

$$
v^{\prime}(a)=\left(a^{2} \log a^{-1}\right)^{-1} .
$$

Our next theorem shows that the above formal considerations do lead to a correct determination of $m(t)$. 
THEOREM V. Let $v(t)$ be a function satisfying (21), $0<a<1$, with the constant of integration chosen so that

$$
v(a)=-S^{\prime \prime}(0)-a^{-1} \log \log a^{-1}+\int_{a}^{1} t^{-2} \log \log t^{-1} d t .
$$

Let $\alpha(t), \beta(t), \gamma(t)$ be defined by (20) for $0<a<1$ with the constant of integration in $\beta(t)$ chosen so that

$$
\beta(a)=\log \log a^{-1}+\int_{0}^{a}\left[v(t)+\left(t \log t^{-1}\right)^{-1}\right] d t .
$$

Then, (2), (3), and (14) are satisfied and

$$
\beta(1)=\lim _{t / 1} \beta(t), \quad \gamma(1)=\lim _{t / 1} \gamma(t)
$$

exist and are finite. Let $(E(t, z))$ be the corresponding family of entire functions satisfying (1), $0<t \leqq 1$, such that (6) and (15) are satisfied. Then, (13) holds for all complex $z$.

In Theorem $\mathrm{V}$, we do not define $m(t)$ for $t>1$. This might be done in a numbers of ways. For instance, we might take

$$
\alpha(t)=\alpha(1)+t-1, \quad \beta(t)=\beta(1), \quad \gamma(t)=\gamma(1)+t-1
$$

so that (4) is satisfied. The corresponding measure $\mu$, defined as in Theorem $\mathrm{I}$, is absolutely continuous with the density

$$
\mu^{\prime}(t)=|E(1, t)|^{-2} \text {. }
$$

It is interesting to write out the assertion of Theorem III explicitly in the situation of Theorem V. Because (20) holds, it takes an especially simple form. The formula

$$
(f(t), g(t)) \rightarrow f(t)+g(t) v(t)
$$

defines a linear isometric transformation of $L^{2}(m)$ onto $L^{2}(0,1)$. Since there are no singular points for this choice of $m(t), L_{0}^{2}(m)$ can be replaced by $L^{2}(0,1)$ in Theorem III, which now has this interpretation for parts A and B.

TheOREM VI. Let $m(t), E(t, z)$, and $v(t)$ be as in Theorem V.

(A) Then,

$$
\phi(t, w)=A(t, w)+B(t, w) v(t)
$$

belongs to $L^{2}(0,1)$ as a function of $t$ for every complex number w. For each element $f(t)$ of $L^{2}(0,1)$, define a corresponding eigentransform $F(z)$ by

$$
\pi F(w)=\int f(t) \phi(t, w) d t
$$


for all complex w. Then, $F(z)$ is an entire function, it belongs to $\mathfrak{H C}(E(1))$, and

$$
\pi \int|F(t)|^{2}|E(1, t)|^{-2} d t=\int|f(t)|^{2} d t
$$

If $G(z)$ is in $\mathcal{H C}(E(1))$, then $G(z)$ is equal to $F(z)$ for some such choice of $f(t)$. If $F(z)$ is the eigentransform of $f(t)$, then $F^{*}(z)$ is the eigentransform of $\bar{f}(t)$.

(B) Let $f(t)$ be an element of $L^{2}(0,1)$ and let $F(z)$ be its eigentransform. $A$ necessary and sufficient condition that $f(t)$ be (equivalent to) an absolutely continuous function and that for some $g(t)$ in $L^{2}(0,1)$

$$
f^{\prime}(a)=v^{\prime}(a) \int_{0}^{a} g(t) d t
$$

for $0<a<1$ and that

$$
f^{\prime}(t)=o\left(v^{\prime}(t)\right), \quad f(t) v^{\prime}(t)-v(t) f^{\prime}(t)=o\left(v^{\prime}(t)\right)
$$

as $t \rightarrow 0$, is that $z F(z)$ belong to $\mathfrak{H C}(E(1))$. In this case, let $G(z)$ be the eigentransform of $g(t)$. Then, $G(z)=z F(z)$ for all complex $z$.

We have seen that when $E(z)=S^{\prime}(z)-i S(z)$, the function $z F(z+1)$ belongs to $\mathfrak{H C}(E)$ whenever $F(z)$ belongs to $\mathfrak{H C}(E)$ and that it has the same norm as $F(z)$. It is interesting to see what the transformation $F(z) \rightarrow z F(z+1)$ corresponds to in $L^{2}(0,1)$.

THEOREM VII. If $f(t)$ is in $L^{2}(0,1)$ and if $\int f(t) d t=0$, then

$$
g(t)=f(t)-t^{-1} \int_{0}^{t} f(s) d s
$$

is in $L^{2}(0,1)$ and has the same norm as $f(t)$. Let $F(z)$ and $G(z)$ be the corresponding eigentransforms, as in Theorem VI. Then, $F(z)=z G(z+1)$ for all complex $z$.

Another way to get a deeper and better understanding of the theorems at the beginning of this paper is to give applications. One way to do this is to take well-known properties of the Fourier transform and look for generalizations in this new context. The Hilbert transform is a close relative of the Fourier transform and it serves our purpose admirably. If $F(x)$ belongs to $L^{2}(-\infty, \infty)$, the Hilbert transform $G(x)$ is defined by

$$
G(x)=P \int \frac{F(t)}{\pi(t-x)} d t,
$$

according to Titchmarsh $[14$, Chapter V], where $P$ denotes a principal value at $t=x$. The function $G(x)$ is defined for almost all real $x$, it belongs to $L^{2}(-\infty, \infty)$, and 


$$
\int|G(t)|^{2} d t=\int|F(t)|^{2} d t
$$

and

$$
F(x)=-P \int \frac{G(t)}{\pi(t-x)} d t
$$

for almost all real $x$. In order to motivate a generalization, we will write (23) formally as

$$
\pi G(x)=\int \frac{F(t)-F(x)}{t-x} d t .
$$

Let $\mu$ be a non-negative measure on the Borel sets of the real line, such that

$$
\int\left(1+t^{2}\right)^{-1} d \mu(t)<\infty
$$

If $F(x)$ belongs to $L^{2}(\mu)$, we would like to define a function $G(x)$ formally by

$$
\pi G(x)=\int \frac{F(t)-F(x)}{t-x} d \mu(t) .
$$

In most cases, the integral occurring in (25) is not absolutely convergent. Even if it is, we will not necessarily agree with (25) in our actual definition, which is based on an example. Since $\mu$ satisfies (24), the function $F(x)$ $=(x-w)^{-1}$ belongs to $L^{2}(\mu)$ if $w$ is not real. In this case,

$$
\frac{F(t)-F(x)}{t-x}=-\frac{F(x)}{(t-w)}
$$

and we should interpret $G(x)$ as

$$
G(x)=-i \phi(w) F(x)
$$

where $\phi(w)$ is a suitable interpretation of the integral

$$
\phi(w)=\frac{1}{\pi i} \int \frac{d \mu(t)}{(t-w)} .
$$

By this, we will mean the choice of a function $\phi(z)$, defined and analytic in the complement of the real axis, such that $\phi(\bar{z})=-\bar{\phi}(z)$, and

$$
\frac{y}{\pi} \int \frac{d \mu(t)}{(t-x)^{2}+y^{2}}=\operatorname{Re} \phi(z)
$$

when $z$ is not real. After partial fraction decompositions, this requirement becomes equivalent to the formula 


$$
\frac{i(\bar{w}-z)}{\pi} \int \frac{d \mu(t)}{(t-z)(t-\bar{w})}=\phi(z)+\phi(w)
$$

whenever $z$ and $w$ are not real. In the case $d \mu=d x$, this interpretation becomes consistent with the ordinary Hilbert transform on choosing $\phi(z)=1$ for $y>0$.

Before completing the definition of our generalized Hilbert transform, we must observe than an important property of the ordinary Hilbert transform is its isometric nature. The generalized Hilbert transform will be an isometric transformation of $L^{2}(\mu)$ into $L^{2}(\nu)$ if $\nu$ is a suitably chosen measure. The right choice of $\nu$ is such that

$$
\frac{y}{\pi} \int \frac{d \nu(t)}{(t-x)^{2}+y^{2}}=\operatorname{Re} \phi(z)^{-1}
$$

whenever $z$ is not real. The existence of such a measure follows by the Poisson representation of a function positive and harmonic in a half-plane if $\phi(z)$ is chosen so that

$$
\phi(i y)^{-1}=o(y)
$$

as $y \rightarrow+\infty$. Then, when $z$ and $w$ are not real,

$$
\frac{i(\bar{w}-z)}{\pi} \int \frac{d \nu(t)}{(t-z)(t-\bar{w})}=\phi(z)^{-1}+\phi(w)^{-1}
$$

and hence by (27)

$$
\int \frac{\phi(z) \Phi(w)}{(t-z)(t-\bar{w})} d \nu(t)=\int \frac{d \mu(t)}{(t-z)(t-\bar{w})} .
$$

We may now interpret (25) in this way. Let $G(x)$ be the unique element of $L^{2}(\nu)$ such that

$$
\int \frac{G(t) d \nu(t)}{t-w}=i \phi(w)^{-1} \int \frac{F(t) d \mu(t)}{t-w}
$$

whenever $w$ is not real.

THeOREM VIII. Let $\mu$ and $\nu$ be non-negative measures on the Borel sets of the real line which satisfy (24), (26), and (28) for some choice of a function $\phi(z)$, defined and analytic when $z$ is not real, such that $\phi(\bar{z})=-\Phi(z)$. If $F(x)$ belongs to $L^{2}(\mu)$, then (31) defines a unique element $G(x)$ of $L^{2}(\nu)$ and

$$
\int|G(t)|^{2} d \nu(t)=\int|F(t)|^{2} d \mu(t)
$$

To see the relevance of the generalized Hilbert transform for Hilbert spaces of entire functions, we must consider another transformation. Let 


$$
M(z)=\left(\begin{array}{ll}
A(z) & B(z) \\
C(z) & D(z)
\end{array}\right)
$$

be a matrix of entire functions which are real for real $z$ and satisfy

$$
\begin{aligned}
A(z) D(z)-B(z) C(z) & =1, \\
\operatorname{Re}[A(z) \bar{D}(z)-B(z) \bar{C}(z)] & \geqq 1, \\
{[B(z) \bar{A}(z)-A(z) \bar{B}(z)] /(z-\bar{z}) } & \geqq 0, \\
{[D(z) \bar{C}(z)-C(z) \bar{D}(z)] /(z-\bar{z}) } & \geqq 0 .
\end{aligned}
$$

We are particularly interested in the case that $A(z)-i B(z)$ satisfies (1) and

$$
A(i y)-i B(i y)=o[D(i y)+i C(i y)]
$$

as $y \rightarrow+\infty$. In this case, $D(z)+i C(z)$ satisfies (1). The desired transformation is defined in this way.

THEOREM IX. Let $M(z)$ be a matrix valued entire function which satisfies (33) and suppose that $A(z)-i B(z)$ satisfies (1) and that (34) holds. Then, for every complex number $w$,

$$
L(w, z)=\frac{1-A(z) \bar{D}(w)+B(z) \bar{C}(w)}{\pi(z-w)}
$$

belongs to $\mathfrak{H C}(A-i B)$ as a function of $z$. If $F(z)$ is in $\operatorname{FC}(A-i B)$, the entire function $G(z)$ defined by

$$
G(w)=\langle F(t), L(w, t)\rangle_{A-i B}
$$

for all complex $w$, belongs to $\operatorname{He}(D+i C)$ and

$$
\|G\|_{D+i C}^{2}=\|F\|_{A-i B}^{2} .
$$

To see the relationship between this last transformation and the generalized Hilbert transform, we must consider a non-negative measure $\mu$ on the Borel sets of the real line such that $\mathfrak{H C}(A-i B)$ is contained isometrically in $L^{2}(\mu)$. Then, by Theorem VA of [8],

$$
\text { 7) } \frac{y}{\pi} \int \frac{|A(t)-i B(t)|^{2} d \mu(t)}{(t-x)^{2}+y^{2}}=\operatorname{Re} \frac{[A(z)-i B(z)]+[A(z)+i B(z)] W(z)}{[A(z)-i B(z)]-[A(z)+i B(z)] W(z)}
$$

for $y>0$, where $W(z)$ is a function which is defined and analytic for $y>0$ and $|W(z)| \leqq 1$. By the proof of Theorem VI of [8], formula (26) holds with

$$
\phi(z)=\frac{[D(z)+i C(z)]+[D(z)-i C(z)] W(z)}{[A(z)-i B(z)]-[A(z)+i B(z)] W(z)}
$$

for $y>0$. If now it is possible to define a non-negative measure $\nu$ on the Borel 
sets of the real line by (28), the proof of Theorem VI of [8] suggests the formula

$$
\frac{y}{\pi} \int \frac{|D(t)+i C(t)|^{2} d \nu(t)}{(t-x)^{2}+y^{2}}=\operatorname{Re} \frac{[D(z)+i C(z)]-[D(z)-i C(z)] W(z)}{[D(z)+i C(z)]+[D(z)-i C(z)] W(z)}
$$

for $y>0$. In fact, the formula always holds if the elements $F(z)$ of $\mathfrak{F C}(D+i C)$, whose products by $z$ belong to this space, are dense in this space. The relationship to the generalized Hilbert transform may now be expressed in this way.

THEOREM X. In the situation of Theorem IX, let $\mu$ and $\nu$ be non-negative measures on the Borel sets of the real line such that (37) and (39) hold for some function $W(z)$, defined and analytic for $y>0$, such that $|W(z)| \leqq 1$. Then, $\operatorname{HC}(A-i B)$ is contained isometrically in $L^{2}(\mu)$, and $\mathfrak{H C}(D+i C)$ is contained isometrically in $L^{2}(\nu)$. Formulas (26) and (28) hold with $\phi(z)$ defined by (38) for $y>0$ and by $\phi(\bar{z})=-\bar{\phi}(z)$ for nonreal values of $z$. Let $F(z)$ belong to $\operatorname{HC}(A-i B)$ and let $G(z)$ be the corresponding element of $\mathfrak{F}(D+i C)$ defined by (35). Then, (31) holds.

At this point we should say something about the formal definition (25) of the generalized Hilbert transform. Whereas this formal definition is often meaningless and may not coincide with the actual definition when it (the formal definition) has meaning, it does nevertheless suggest two things which are entirely correct.

Theorem XI. In the situation of Theorem IX, if $F(z)$ is in $\mathfrak{F C}(A-i B)$, then

$$
[F(z)-F(w)] /(z-w)
$$

belongs to $\mathfrak{H C}(A-i B)$ as a function of $z$ for every complex number w. If $G(z)$ is defined by (35), then

$$
G^{*}(w)=\left\langle F^{*}(t), L(w, t)\right\rangle_{A-i B}
$$

and

$$
\frac{G(z)-G(w)}{z-w}=\left\langle\frac{F(t)-F(w)}{t-w}, L(z, t)\right\rangle_{A-i B}
$$

for all complex $z$.

Our reason for bringing up the generalized Hilbert transform at this time is to describe its relationship to the generalized Fourier transform of Theorem III. To do this, we must consider a matrix valued function $m(t)$ of $t>0$ which satisfies (2), (3), (4), and

$$
\gamma(t)>0 \text { for } t>0 \text { and } \lim _{t \searrow 0} \gamma(t)=0 .
$$


For each complex number $w$, let $M(t, w)$ be the unique continuous matrix valued function of $t \geqq 0$ such that

$$
M(a, w) I-I=w \int_{0}^{a} M(t, w) d m(t)
$$

for $a \geqq 0$. Then, for each fixed $a \geqq 0$,

$$
M(a, z)=\left(\begin{array}{ll}
A(a, z) & B(a, z) \\
C(a, z) & D(a, z)
\end{array}\right)
$$

is a matrix valued entire function of $z$ which satisfies (33) by Theorem VI of [10]. When $a>0, A(a, z)-i B(a, z)$ and $D(a, z)+i C(a, z)$ satisfy (1) and (34) and

$$
D(a, i y)+i C(a, i y)=o[A(a, i y)-i B(a, i y)]
$$

as $y \rightarrow \infty$ because of (40) and Lemmas 9 and 10 of [10]. By the proof of Theorem VIII of [10], there exist unique non-negative measures $\mu$ and $\nu$ on the Borel sets of the real line such that for each regular $a>0$

$$
\begin{aligned}
& \frac{y}{\pi} \int \frac{|A(a, t)-i B(a, t)|^{2} d \mu(t)}{(t-x)^{2}+y^{2}} \\
& =\operatorname{Re} \frac{[A(a, z)-i B(a, z)]+[A(a, z)+i B(a, z)] W(a, z)}{[A(a, z)-i B(a, z)]-[A(a, z)+i B(a, z)] W(a, z)}
\end{aligned}
$$

and

$$
\begin{aligned}
& \frac{y}{\pi} \int \frac{|D(a, t)+i C(a, t)|^{2} d \mu(t)}{(t-x)^{2}+y^{2}} \\
& =\operatorname{Re} \frac{[D(a, z)+i C(a, z)]-[D(a, z)-i C(a, z)] W(a, z)}{[D(a, z)+i C(a, z)]+[D(a, z)-i C(a, z)] W(a, z)}
\end{aligned}
$$

for $y>0$, where $W(a, z)$ is a function analytic for $y>0$ and bounded by 1 , defined as in Lemma 1 below. We are in the situation of Theorem $\mathrm{X}$ for every regular $a>0$.

THEOREM XII. Let $m(t)$ be a matrix valued function of $t>0$ which satisfies (2), (3), (4), and (40). Let $M(t, z)$ be defined by (41) and let $\mu$ and $\nu$ be the measures defined by (43) and (44).

(A) Let $a>0$ be regular with respect to $m(t)$ and let $(f, g)$ be an element of $L_{0}^{2}(m)$ which is equivalent to zero outside of $[0, c]$. Let $F(z)$ and $G(z)$ be the corresponding entire functions defined by (9) and

$$
\pi G(w)=\int(f(t), g(t)) d m(t)(C(t, \bar{w}), D(t, \bar{w}))^{-}
$$


for all complex $w$. Then, $F(z)$ belongs to $\mathfrak{H C}(A(a)-i B(a))$ and (35) holds with the inner product taken in this Hilbert space and with $L(w, z)=L(a, w, z)$.

(B) Let $(f, g)$ be an element of $L_{0}^{2}(m)$, let $F(x)$ be the corresponding element of $L^{2}(\mu)$ defined by (11), and let $G(x)$ be defined analogously by

$$
G(x)=\lim _{a \rightarrow \infty} \int_{0}^{a}(f(t), g(t)) d m(t)(C(t, x), D(t, x))^{-}
$$

with convergence in the metric of $L^{2}(\nu)$. Then, (31) holds.

Since Theorem XII involves a complicated apparatus, we will describe its effect in the Fourier case which induced the generalization. Then,

$$
\begin{gathered}
\alpha(t)=t, \quad \beta(t)=0, \quad \gamma(t)=t, \\
A(t, z)=\cos (t z), \quad B(t, z)=\sin (t z), \\
C(t, z)=-\sin (t z), \quad D(t, z)=\cos (t z), \\
d \mu=d t=d \nu, \quad W(a, z)=0, \\
\phi(x+i y)=\operatorname{sgn} y .
\end{gathered}
$$

Every number $c>0$ is regular for this choice of $m(t)$. The Hilbert space $L_{0}^{2}(m)$ consists of (equivalence classes of) pairs $(f, g)$ of measurable complex valued functions $f(t), g(t)$ of $t \geqq 0$ such that

$$
\|(f, g)\|^{2}=\int|f(t)|^{2} d t+\int|g(t)|^{2} d t .
$$

If $f(t)$ and $g(t)$ vanish a.e. for $t>a$, formula (9) reduces to

$$
\pi F(z)=\int f(t) \cos (t z) d t+\int g(t) \sin (t z) d t
$$

whereas (45) reads

$$
\pi G(z)=-\int f(t) \sin (t z) d t+\int g(t) \cos (t z) d t
$$

Formula (31) becomes

$$
\int \frac{G(t) d t}{t-w}=i \int \frac{F(t) d t}{t-w}
$$

when $w$ is in the upper half-plane; the sign must be reversed in the lower half-plane. Formula (35) is

$$
G(w)=\int F(t) \frac{1-\cos a(t-w)}{\pi(t-w)} d t .
$$


These are familiar formulas in the theory of the Hilbert transform.

It is convenient to break up the proofs by using several lemmas. The first two are closely related to Theorems XI and XII of [10]. The fifth lemma is a variation of the Stone-Weierstrass theorem. Only minor changes need be made in the proof of [4] to get this result, and such an approach seems preferable to quoting more elaborate theorems. Perhaps the fourth lemma has a separate interest, and it also could be proved by using more elaborate techniques of functional analysis. The other lemmas are just minor calculations which we wish to separate from the essential parts of the proofs.

Leммa 1. Let $m(t)$ be a matrix valued function of $t>0$ which satisfies (2), (3), and (4). Then, for each $a>0$ and each complex number w, there exists $a$ unique continuous matrix valued function

$$
M(a, t, w)=\left(\begin{array}{ll}
A(a, t, w) & B(a, t, w) \\
C(a, t, w) & D(a, t, w)
\end{array}\right)
$$

of $t \geqq a$ such that

$$
M(a, b, w) I-I=w \int_{a}^{b} M(a, t, w) d m(t)
$$

for $b \geqq a$. For each fixed $a$ and $b, M(a, b, z)$ is a matrix valued entire function of $z$ which satisfies (33). For each $a>0$, there is a unique function $W(a, z)$, defined and analytic for $y>0$, such that $|W(a, z)| \leqq 1$, and

$$
\frac{1+W(a, z)}{1-W(a, z)}=\lim _{c \rightarrow \infty} \frac{D(a, c, z)+i C(a, c, z)}{A(a, c, z)-i B(a, c, z)}
$$

for $y>0$.

In Lemma 1 , the possibility that $W(a, z)=1$ identically is not excluded. In this case, (48) is to be interpreted as convergence on the Riemann sphere. Equivalently, the limit obtained by solving formally for $W(a, z)$ is valid in the ordinary sense.

LEMMA 2. Let $W(z)$ be defined and analytic for $y>0$ and such that $|W(z)|$ $\leqq 1$. Let $a>0$. Then, there exists a matrix valued function $m(t)$, as in Lemma 1 , such that

$$
W(z)=W(a, z)
$$

for $y>0$.

LEMMA 3. Let $E(z)$ be an entire function satisfying (1). Then, there exists a sequence $\left(E_{n}(z)\right)$ of polynomials, satisfying (1), with no real zeros, such that

$$
E^{*}(z) / E(z)=\lim E_{n}^{*}(z) / E_{n}(z)
$$

for $y>0$. 
LEMMA 4. Let $E(z)$ be an entire function, satisfying (1), with no real zeros. Suppose that $\mathfrak{H C}(E)$ does not have dimension 1 . Then, there is an entire function $E(b, z)$, satisfying (1), with no real zeros, such that $\mathfrak{H C}(E(b))$ is contained isometrically in $\mathfrak{H C}(E)$ and is a proper subspace of $\mathcal{H C}(E)$.

Lemma 5. Let $[a, b]$ be a finite interval and let $B$ be a vector space of pairs $(f(t), g(t))$ of continuous complex valued functions on $[a, b]$. We make three hypotheses.

(a) The pair $(\bar{f}(t), \bar{g}(t))$ belongs to $B$ whenever $(f(t), g(t))$ belongs to $B$.

(b) The pair $h(t)(f(t), g(t))$ belongs to $B$ whenever $(f(t), g(t))$ belongs to $B$ and $h(t)$ is a continuous complex valued function of $t$ in $[a, b]$.

(c) For each $x$ in $[a, b]$ and each pair $(u, v)$ of complex numbers, there is an element $(f(t), g(t))$ of $B$ such that

$$
(u, v)=(f(x), g(x)) .
$$

Then, if $(f(t), g(t))$ is any pair of continuous complex valued functions on $[a, b]$, there is a sequence $\left(f_{n}(t), g_{n}(t)\right)$ of elements of $B$ such that

$$
(f(t), g(t))=\lim \left(f_{n}(t), g_{n}(t)\right)
$$

uniformly in $[a, b]$.

Lемма 6. In the situation of Theorem I,

$$
\pi K(b, w, z)-\pi K(a, w, z)
$$

$$
=\int_{a}^{b}(A(t, z), B(t, z)) d m(t)(A(t, w), B(t, w))^{-}
$$

for all complex $z$ and when $0<a<b<\infty$.

Lemma 7. Let $w_{0}$ be a complex number with $i\left(\bar{w}_{0}-w_{0}\right)>0$ and let $A_{0}$ and $B_{0}$ be complex numbers such that

$$
\left|A_{0}+i B_{0}\right|<\left|A_{0}-i B_{0}\right| \text {. }
$$

Then, there exists a unique linear function $E(z)$ satisfying (1) such that

$$
A\left(w_{0}\right)=A_{0} \text { and } B\left(w_{0}\right)=B_{0} .
$$

Proof of Lemma 1. The existence and uniqueness of $M(a, t, w)$ is Theorem VI of [10] with a change of variable. It is shown there that $M(a, b, z)$ is a matrix valued entire function of $z$ which satisfies (33). If $\alpha(t)=\alpha(a)$ for $t>a$, then $\beta(t)=\beta(a)$ for $t>a$ by (2). Then,

$$
\begin{array}{ll}
A(a, t, z)=1, & B(a, t, z)=0, \\
C(a, t, z)=\gamma(a) z-\gamma(t) z, & D(a, t, z)=1 .
\end{array}
$$

In this case, $W(a, z)=1$ identically because of (4). Otherwise, the existence of $W(a, z)$ follows as in the proof of Theorem VIII of [10]. This is based on 
Lemma 9 of $[10]$ and depends on the fact that

$$
i A(a, c, z) \bar{B}(a, c, z)-i B(a, c, z) \bar{A}(a, c, z) \rightarrow \infty
$$

as $c \rightarrow \infty$ when $y>0$, and $a$ and $z$ are held fixed. If $\alpha(t)>\alpha(a)$ for $t>a$, this follows from (4) and Theorem VIII of [10]. Otherwise, there is a largest number $b$ such that $\alpha(b)=\alpha(a)$. Then,

$$
A(a, c, z)=A(b, c, z), \quad B(a, c, z)=B(b, c, z)
$$

and $\alpha(t)>\alpha(b)$ for $t>b$. In this case also, (51) follows from (4) and Theorem VIII of $[10]$.

Proof of Lemma 2. If $W(z)=1$ identically, we may take $\alpha(t)=t$ for $t \leqq a$, $\alpha(t)=\alpha(a)$ for $t \geqq a, \beta(t)=0, \gamma(t)=t$. Otherwise, the hypotheses imply that

$$
[1+W(z)] /[1-W(z)]
$$

is defined and analytic for $y>0$ and has a non-negative real part. By the Poisson representation of a function positive and harmonic in a half-plane, there is a non-negative measure $\mu$ on the Borel sets of the real line and a number $p \geqq 0$ such that

$$
\operatorname{Re} \frac{1+W(z)}{1-W(z)}=\frac{y}{\pi} \int \frac{d \mu(t)}{(t-x)^{2}+y^{2}}+p y
$$

for $y>0$. Let $b>a$. Let $m_{0}(t)$ be defined linearly in $[a, b]$ so that

$$
\alpha_{0}(b)=\alpha_{0}(a), \quad \beta_{0}(b)=\beta_{0}(a), \quad \gamma_{0}(b)=\gamma_{0}(a)+p .
$$

If $\mu$ vanishes identically, let

$$
\alpha_{0}(t)=t-b+\alpha_{0}(b), \quad \beta_{0}(t)=\beta_{0}(b), \quad \gamma_{0}(t)=\gamma_{0}(b),
$$

for $t \geqq b$. If $\mu$ does not vanish identically, we apply Theorem XII of [10]. There is an absolutely continuous matrix valued function $m_{0}(t)$ of $t \geqq b$, satisfying (2) for $t \geqq b$ and (4), such that

$$
\frac{y}{\pi} \int \frac{d \mu(t)}{(t-x)^{2}+y^{2}}=\operatorname{Re} \lim _{c \rightarrow \infty} i C_{0}(b, c, z) / A_{0}(b, c, z)
$$

for $y>0$, where $M_{0}(b, t, z)$ is defined by $(47)$ for $m(t)=m_{0}(t)$ for $t \geqq b$. The function given by Theorem XII of [10] can be altered by a constant without changing its relevant properties. It is to be determined so that $m_{0}(t)$ has the previously defined value when $t=b$. Since

$$
\begin{array}{ll}
A_{0}(a, b, z)=1, & B_{0}(a, b, z)=0, \\
C_{0}(a, b, z)=-p z, & D_{0}(a, b, z)=1,
\end{array}
$$

we have

$$
\operatorname{Re}[1+W(z)] /[1-W(z)]=\operatorname{Re} \lim _{c \rightarrow \infty} i C_{0}(a, c, z) / A_{0}(a, c, z)
$$


for $y>0$. Since (51) holds, we see by Lemma 9 of [10] that

$$
\operatorname{Re}[1+W(z)] /[1-W(z)]=\operatorname{Re}\left[1+W_{0}(a, z)\right] /\left[1-W_{0}(a, z)\right]
$$

for $y>0$, where $W_{0}(a, z)$ is defined by $(48)$ for $m_{0}(t)$. By the Cauchy-Riemann equations, there is a real number $q$ such that

$$
[1+W(z)] /[1-W(z)]=\left[1+W_{0}(a, z)\right] /\left[1-W_{0}(a, z)\right]+i q
$$

for $y>0$. The lemma follows on defining

for $t \geqq a$.

$$
\begin{aligned}
& \alpha(t)=\alpha_{0}(t), \\
& \beta(t)=\beta_{0}(t)+q \alpha_{0}(t), \\
& \gamma(t)=\gamma_{0}(t)+2 q \beta_{0}(t)+q^{2} \gamma_{0}(t)
\end{aligned}
$$

Proof of Lemma 3. Since $E^{*}(z) / E(z)$ is defined and analytic for $y>0$, is bounded by 1 , is analytic across the real axis, and has absolute value 1 on the real axis,

$$
E^{*}(z) / E(z)=\exp (2 i a z-2 i c) \prod\left[\left(1-z / w_{n}\right) /\left(1-z / \bar{w}_{n}\right)\right]
$$

where $a \geqq 0, c$ is real, $i\left(\bar{w}_{n}-w_{n}\right)>0$ for every $n$,

$$
\sum i\left(\bar{w}_{n}-w_{n}\right)\left|w_{n}\right|^{-2}<\infty,
$$

and $\left|w_{n}\right| \rightarrow \infty$ if the number of these zeros is infinite. See Boas [1, p. 92]. Let

$$
E_{n}(z)=e^{i c}(1-i a z / n)^{n} \prod_{1}^{n}\left(z-\bar{w}_{k}\right)
$$

if the number of zeros is infinite. Otherwise, let

$$
E_{n}(z)=e^{i c}(1-i a z / n)^{n} \prod\left(z-\bar{w}_{k}\right) .
$$

Proof of Lemma 4. Let $\left(E_{n}(z)\right)$ be a sequence of polynomials chosen for $E(z)$ as in Lemma 3. By multiplying each one by a constant, we may suppose that $E_{n}(0)=1$. By Theorem $\mathrm{V}$ of $[10]$, there is a matrix valued function $m_{n}(t)$ of $t>0$, satisfying (2), (3), and (4), and a corresponding family $\left(E_{n}(t, z)\right)$ of entire functions, satisfying (1), with these properties: for each complex number $w, E_{n}(t, w)$ is a continuous function of $t>0$ and (6) holds for $m(t)$ $=m_{n}(t)$, and (7) is true, and $E_{n}(z)=E_{n}\left(c_{n}, z\right)$ for some $c_{n}>0$. By a reparametrization, we may suppose that $c_{n}=c$ is independent of $n$. Since each $E_{n}(z)$ is a polynomial, the proof of Theorem $\mathrm{V}$ of [10] shows that

$$
\gamma_{n}(0)=\lim _{t \searrow 0} \gamma_{n}(t)
$$

is finite for every $n$. We cannot have

$$
\alpha_{n}(c)+\gamma_{n}(c)-\alpha_{n}(0)-\gamma_{n}(0)
$$


converge to 0 as $n \rightarrow \infty$, for then Theorem IX of [10] would imply that $E_{n}(z)$ $\rightarrow 1$ for all complex $z$, and hence that

$$
E_{n}^{*}(z) / E_{n}(z) \rightarrow 1
$$

for $y>0$; by (49) this contradicts the hypothesis that $E(z)$ satisfies (1). By considering subsequences, we see similarly that (52) is bounded away from zero. Choose $a>0$ so that $a<c$ and (52) is greater than $c-a$ for every $n$. By reparametrizations, we may suppose that

$$
\alpha_{n}(t)+\gamma_{n}(t)=t
$$

for $t \geqq a$, and that $\beta_{n}(c)=0$. As in the proof of Theorem $\mathrm{V}$ of [10], we may suppose, by passing to a subsequence, that

$$
m(t)=\lim m_{n}(t)
$$

converges uniformly on every bounded subset of $[a, \infty)$. Therefore,

$$
m(t)=\left(\begin{array}{ll}
\alpha(t) & \beta(t) \\
\beta(t) & \gamma(t)
\end{array}\right)
$$

is defined for $t \geqq a$, and $\alpha(t), \beta(t), \gamma(t)$ are real valued, absolutely continuous functions which satisfy (2) and

$$
\alpha(t)+\gamma(t)=t .
$$

When $t \geqq b \geqq a$, let $M_{n}(b, t, z)$ and $M(b, t, z)$ be defined as in Lemma 1 for $m_{n}(t)$ and $m(t)$, respectively. As in the proof of Theorem IV of [10],

$$
\left(A_{n}(t, z), B_{n}(t, z)\right)=\left(A_{n}(b, z), B_{n}(b, z)\right) M_{n}(b, t, z)
$$

for all complex $z$. Let $A(b, z)$ and $B(b, z)$ be the unique entire functions of $z$ such that

$$
(A(z), B(z))=(A(b, z), B(b, z)) M(b, c, z)
$$

when $a \leqq b \leqq c$. By (33), these entire functions are real for real $z$. By the proof of Theorem XII of [10],

$$
M(b, c, z)=\lim M_{n}(b, c, z)
$$

for all complex z. By (49) and (53),

$$
Z(b, z)=\lim E_{n}^{*}(b, z) / E_{n}(b, z)
$$

exists for $y>0$, and $|Z(b, z)| \leqq 1$, and

$$
A(b, z)+i B(b, z)=[A(b, z)-i B(b, z)] Z(b, z) .
$$

Since $M(b, c, z)$ satisfies (33) and $E(z)$ has no zeros for $y \geqq 0$ by hypothesis, it follows that 


$$
E(b, z)=A(b, z)-i B(b, z)
$$

has no zeros for $y \geqq 0$ and that

$$
|E(b, \bar{z})| \leqq|E(b, z)|
$$

for these values of $z$. Either $E(b, z)$ satisfies (1) or

$$
E(b, z) E^{*}(b, w)=E^{*}(b, z) E(b, w)
$$

for all complex $z$ and $w$. By the proof of Theorem IV of [10], if $a<b<c$ and if $b$ is regular with respect to $m(t), E(b, z)$ satisfies (1) and $\Re C(E(b))$ is contained isometrically in $\mathfrak{H}(E)$. Since $m(t)$ is not a constant in $[b, c]$ by construction, $\mathcal{F}(E(b))$ is then a proper subspace of $\mathcal{F}(E)$. It remains to consider the case in which the interval $(a, c)$ contains only singular points with respect to $m(t)$. Then,

$$
\begin{array}{ll}
A(a, c, z)=1+\beta(a) z-\beta(c) z, & B(a, c, z)=\alpha(c) z-\alpha(a) z, \\
C(a, c, z)=\gamma(a) z-\gamma(c) z, & D(a, c, z)=1+\beta(c) z-\beta(a) z,
\end{array}
$$

for all complex $z$ and

$$
[\beta(c)-\beta(a)]^{2}=[\alpha(c)-\alpha(a)][\gamma(c)-\gamma(a)]
$$

and

$$
\alpha(c)-\alpha(a)+\gamma(c)-\gamma(a)=c-a>0 .
$$

Let $u$ and $v$ be a choice of complex numbers such that

and let

$$
\begin{aligned}
& \alpha(c)-\alpha(a)=\pi u \bar{u} \\
& \beta(c)-\beta(a)=\pi u \bar{v} \\
& \gamma(c)-\gamma(a)=\pi v \bar{v}
\end{aligned}
$$

$$
G(z)=A(a, z) u+B(a, z) v=A(z) u+B(z) v .
$$

If $E(a, z)$ satisfies $(54)$, it follows that

$$
K(w, z)=G(z) \bar{G}(w)
$$

and that $G(z)$ is an element of norm 1 in $\mathfrak{H C}(E)$, which spans $\mathfrak{H C}(E)$; our hypotheses exclude this case. Therefore, $E(a, z)$ satisfies $(1)$ and

$$
K(w, z)=K(a, w, z)+G(z) \bar{G}(w)
$$

for all complex $z$ and $w$. Since $K(a, z, z) \geqq 0$ for all complex $z$,

$$
|G(z)|^{2} \leqq K(z, z)
$$

for all complex $z$, and

$$
|G(i y)|=o(E(i|y|))
$$


as $|y| \rightarrow \infty$. By the proof of Lemma 11 of [10], $G(z)$ belongs to $\mathfrak{H}(E)$. We may apply Theorem II of [8]. Since $G(z)$ does not span $\mathfrak{H C}(E)$ by hypothesis and since $E(z)$ has no real zeros, the orthogonal complement of $G(z)$ in $\operatorname{FC}(E)$ satisfies (H1), (H2), and (H3), and for each complex number $w$, there is an element $F(z)$ of $\mathfrak{H C}(E)$ orthogonal to $G(z)$, such that $F(w) \neq 0$. The lemma follows from the theorem of [7] applied to the orthogonal complement of $G(z)$ in $\mathfrak{H C}(E)$.

Proof of Lemma 5. Since the proof is essentially the same as that given in [4] for the Stone-Weierstrass theorem, we only sketch the present proof. The relevant Banach space consists of all pairs $(f(t), g(t))$ of continuous complex valued functions on $[a, b]$, with the norm

$$
\max _{a \leqq t \leqq b} \max (|f(t)|,|g(t)|) \text {. }
$$

The space is essentially the Banach space of all continuous complex valued functions on the cartesian product of $[a, b]$ with a two-point set, with the functions taken in the uniforn norm. A continuous linear functional on this space is of the form

$$
(f, g) \rightarrow \int f(t) d \mu(t)+\int g(t) d \nu(t)
$$

where $\mu$ and $\nu$ are a pair of complex valued measures on the Borel sets of $[a, b]$ such that

$$
\int|d \mu(t)|+\int|d \nu(t)|<\infty
$$

The functionals are considered in the weak topology, and the set of all those for which

$$
\int|d \mu(t)|+\int|d \nu(t)| \leqq 1
$$

is compact. To prove the lemma, we must show that if

$$
\int f(t) d \mu(t)+\int g(t) d \nu(t)=0
$$

for every pair $(f(t), g(t))$ in $\leftrightarrow$, then $\mu$ and $\nu$ must vanish identically. Because of (a), it is sufficient to show this when $\mu$ and $\nu$ are real valued measures. Because of (b) and the Krein-Milman theorem, as used in [4], it is sufficient to consider the case in which $\mu$ and $\nu$ are supported at a single point $x$. The desired conclusion then follows from (c).

Proof of Lemma 6. By hypothesis, for each fixed complex number $w, A(t, w)$ and $B(t, w)$ are absolutely continuous functions of $t>0$ such that 


$$
\begin{aligned}
B^{\prime}(t, w) & =w A(t, w) \alpha^{\prime}(t)+w B(t, w) \beta^{\prime}(t) \\
-A^{\prime}(t, w) & =w A(t, w) \beta^{\prime}(t)+w B(t, w) \gamma^{\prime}(t)
\end{aligned}
$$

a.e. for $t>0$. Therefore, for each fixed $z$ and $w, K(t, w, z)$ is an absolutely continuous function of $t>0$ such that

$$
\begin{aligned}
\pi K^{\prime}(t, w, z)= & A(t, z) \bar{A}(t, w) \alpha^{\prime}(t)+A(t, z) \bar{B}(t, w) \beta^{\prime}(t) \\
& +B(t, z) \bar{A}(t, w) \beta^{\prime}(t)+B(t, z) \bar{B}(t, w) \gamma^{\prime}(t)
\end{aligned}
$$

a.e. for $t>0$, at least when $z \neq \bar{w}$. The desired formula now follows by integrating each side from $t=a$ to $t=b$, when $z \neq \bar{w}$. The case $z=\bar{w}$ can be obtained by continuity. For this, one needs only show that the right hand side of (50) depends continuously on $z$ when $a, b, w$ are held fixed. Let $M(a, t, z)$ be defined as in Lemma 1 for $t \geqq a$, so that

$$
(A(t, z), B(t, z))=(A(a, z), B(a, z)) M(a, t, z)
$$

and use the estimates of $M(a, b, z)$ given by Theorem IX of [10].

Proof of Lemma 7. Since $A(z)$ and $B(z)$ are to be real for real $z, E(z)$ must be chosen so that

$$
E\left(w_{0}\right)=A_{0}-i B_{0}, \quad E\left(\bar{w}_{0}\right)=\bar{A}_{0}-i \bar{B}_{0} .
$$

Since $E(z)$ is to be linear and $\bar{w}_{0} \neq w_{0}$ by hypothesis, it is uniquely determined by these two conditions. All we need do is show that the linear function which satisfies these two conditions also satisfies (1). Obviously, $E(z)$ is not a constant. Therefore, it has a zero $w$. Since

$$
\left|E^{*}\left(w_{0}\right) / E\left(w_{0}\right)\right|=\left|w_{0}-\bar{w}\right| /\left|w_{0}-w\right|<1
$$

by hypothesis and our construction of $E(z)$, and since $i\left(\bar{w}_{0}-w_{0}\right)>0$, it follows that $i(\bar{w}-w)<0$. Therefore,

$$
\left|E^{*}(z) / E(z)\right|=|z-\bar{w}| /|z-w|<1
$$

for $i(\bar{z}-z)>0$, and this implies (1).

Proof of Theorem I. Let $M(a, b, z)$ be defined as in Lemma 1 when $a \leqq b$. By (6) and the uniqueness part of Theorem VI of [10],

$$
(A(b, z), B(b, z))=(A(a, z), B(a, z)) M(a, b, z)
$$

for all complex $z$. When $a$ and $b$ are regular points with respect to $m(t)$, the isometric inclusion of $\mathfrak{H C}(E(a))$ in $\mathfrak{H C}(E(b))$ follows by the proof of Theorem IV of [10]. Since $K(b, \bar{w}, \bar{w})=K(b, w, w)$, it is sufficient to establish (8) when $i(\bar{w}-w)>0$. For this, let $a>0$ be held fixed along with $w$. Choose $E_{0}(a, z)$ by Lemma 7 to be the linear function which satisfies (1) such that

$$
A_{0}(a, w)=A(a, w), B_{0}(a, w)=B(a, w) .
$$

Let 


$$
E_{0}(b, z)=A_{0}(b, z)-i B_{0}(b, z)
$$

where

$$
\left(A_{0}(b, z), B_{0}(b, z)\right)=\left(A_{0}(a, z), B_{0}(a, z)\right) M(a, b, z)
$$

when $b \geqq a$. By the proof of Theorem VII of [8], $E_{0}(b, z)$ satisfies (1). By (55),

$$
A_{0}(b, w)=A(b, w), \quad B_{0}(b, w)=B(b, w) .
$$

By Theorem VIII of [10],

$$
K(b, w, w)=K_{0}(b, w, w)
$$

does go to infinity with $b$. The existence of $\mu$ now follows as in the proof of Theorem VIII of [10], using Lemma 1. The arguments there show that $\mathfrak{H C}(E(a))$ is contained isometrically in $L^{2}(\mu)$ when $a$ is regular, and that the union of these spaces is dense in $L^{2}(\mu)$.

Proof of Theorem II. Since $\mathfrak{H C}(E)$ is contained isometrically in $L^{2}(\mu)$ and $E(z)$ has no real zeros, there is, by Theorem $\mathrm{V}$ of [8], a function $W(z)$, defined and analytic for $y>0$, such that $|W(z)| \leqq 1$, and

$$
\frac{y}{\pi} \int \frac{|E(t)|^{2} d \nu(t)}{(t-x)^{2}+y^{2}}=\operatorname{Re} \frac{E(z)+E^{*}(z) W(z)}{E(z)-E^{*}(z) W(z)}
$$

for $y>0$. Let $c>0$ be chosen arbitrarily and write $E(c, z)=E(z)$. It may be that $\mathfrak{H C}(E(c))$ has dimension 1 . Otherwise, by Lemma 4 , there is an entire function $E(b, z)$, satisfying (1), with no real zeros, such that $\mathfrak{F C}(E(b))$ is contained isometrically in $\mathfrak{H}(E(c))$ and the inclusion is proper. By Theorem III of $[10]$, there is a unique matrix valued entire function $M(b, c, z)$ satisfying (33), such that (55) holds with $b$ replaced by $c$ and $a$ replaced by $b$. By Theorem I of [8], $E(b, z)$ may be chosen so that $M(b, c, 0)=1$. It is convenient to choose the index $b$ a number in $(0, c)$. It may be that $\mathfrak{F C}(E(b))$ has dimension 1. Otherwise, the process may be repeated. It is clear that we may construct inductively a family $(E(t, z))$ of entire functions satisfying (1), with no real zeros, $t$ in $S$, with these properties: the indexing set $S$ is a subset of $(0, c]$ containing $c$; when $a$ and $b$ are in $S$ and $a<b, \mathfrak{F}(E(a))$ is contained isometrically in $\mathcal{F C}(E(b))$ and the inclusion is proper; the unique matrix valued entire function $M(a, b, z)$ satisfying (33) and (55) has the property that $M(a, b, 0)$ $=1$; either the set $S$ is finite and some $\mathfrak{F C}(E(a))$, with $a$ in $S$, has dimension 1 , or else the set $S$ is countably infinite. If $S$ is infinite, consider the intersection $\mathfrak{H C}$ of the spaces $\mathfrak{H C}(E(a))$, with $a$ in $S$. Then, $\mathcal{H C}$ is a Hilbert space of entire functions in the metric of $\mathfrak{F}(E)$. It satisfies the axioms $(\mathrm{H} 1),(\mathrm{H} 2)$, and $(\mathrm{H} 3)$, since this is true of every $\mathfrak{H C}(E(a))$, with $a$ in $S$. If $\mathcal{H C}$ contains a nonzero element, then $\mathcal{H C}$ is equal isometrically to $\mathfrak{H C}(E(s))$ for some entire function $E(s, z)$ satisfying (1). For each $a$ in $S, E(a, z)$ has no real zeros by construction, and so $F(z) /(z-w)$ belongs to $\mathfrak{H C}(E(a))$ whenever $F(z)$ belongs to $\mathfrak{H}(E(a))$ and 
$F(w)=0$, for every complex number $w$. Therefore, $F(z) /(z-w)$ belongs to $\mathfrak{H C}(E(s))$ whenever $F(z)$ belongs to $\mathfrak{H}(E(s))$ and $F(w)=0$, for every complex number $w$. This implies that $E(s, z)$ has no real zeros. It is now clear that the family of entire functions $(E(t, z)), t$ in $S$, can be redefined so as to have this property in addition to the previous ones: if $S$ is an infinite set, the intersection of the spaces $\mathfrak{H}(E(a))$, with $a$ in $S$, is the zero subspace of $\mathfrak{H C}(E)$. By omitting points of $S$ if necessary, we may suppose that the only point of accumulation of $S$ is at the origin. The positive real axis is now the union of intervals of three kinds: (1) the interval $[c, \infty) ;(2)$ intervals $[a, b]$ where $a$ and $b$ are in $S$ and there is no element of $S$ in $(a, b)$; (3) a possible interval $(0, a]$ where $a$ is in $S$ and there is no element of $S$ in $(0, a)$. We will show how to define $m(t)$ in each such interval. Define $m(t)$ in $[c, \infty)$ as in Lemma 1 so that $W(z)=W(c, z)$ for $y>0$. This is possible by Lemma 2. For an interval $[a, b]$ of the form (2), define $m(t)$ so that the unique solution $M(a, t, z)$ of (47) for $a \leqq t \leqq b$ agrees with our previous definition of $M(a, b, z)$ when $t=b$. This is possible by Theorem VII of [10]. In the possible interval $(0, a)$ of the form (3), let

where

$$
\begin{aligned}
& \alpha(t)=\pi u \bar{u} t / a, \\
& \beta(t)=\pi u \bar{v} t / a, \\
& \gamma(t)=\pi v \bar{v} t / a,
\end{aligned}
$$

$$
G(z)=A(a, z) u+B(a, z) v
$$

is an element of norm 1 in $\mathfrak{H C}(E(a))$. This is possible by Theorem I of [10]. The construction is easily seen to have the required properties.

Proof of Theorem IIIA. Since $c>0$ is a regular point by hypothesis, an interval $(a, b)$ of singular points must lie entirely to the left or to the right of $c$. If it lies to the left of $c$,

$$
(A(t, z), B(t, z))=(A(a, z), B(a, z)) M(a, t, z)
$$

for $a \leqq t \leqq b$, where

$$
\begin{array}{ll}
A(a, t, z)=1-\beta(t) z+\beta(a) z, & B(a, t, z)=\alpha(t) z-\alpha(a) z, \\
C(a, t, z)=\gamma(a) z-\gamma(t) z, & D(a, t, z)=1+\beta(t) z-\beta(a) z .
\end{array}
$$

We can show that $(A(t, z), B(t, z))$ is equivalent to a constant in $(a, b)$ by showing that

$$
\begin{aligned}
& (\alpha(t), \beta(t)) I-(\alpha(a), \beta(a)) I, \\
& (\beta(t), \gamma(t)) I-(\beta(a), \gamma(a)) I,
\end{aligned}
$$

is equivalent to zero in $(a, b)$. Since the interval $(a, b)$ contains only singular points, there is a nondecreasing, absolutely continuous function $h(t)$ of $t$ in $(a, b)$ such that 


$$
\begin{aligned}
& \alpha(t)-\alpha(a)=p h(t), \\
& \beta(t)-\beta(a)=q h(t), \\
& \gamma(t)-\gamma(a)=r h(t),
\end{aligned}
$$

where $p, q, r$ are real numbers such that $p \geqq 0, r \geqq 0$, and $p r=q^{2}$. It follows that

$$
\begin{aligned}
& (\alpha(t), \beta(t)) \operatorname{Im}^{\prime}(t) I(\alpha(t), \beta(t))^{-}, \\
& (\beta(t), \gamma(t)) \operatorname{Im}^{\prime}(t) I(\beta(t), \gamma(t))^{-},
\end{aligned}
$$

vanishes a.e. in $(a, b)$. Let $z=w$ in (50) with $b$ replaced by $c$ and $a$ replaced by $\epsilon$, where $\epsilon \searrow 0$. Since $K(\epsilon, w, w) \geqq 0$, it follows that

$$
\chi(c, t)(A(t, w), B(t, w))
$$

belongs to $L_{0}^{2}(m)$. Since

$$
|K(\epsilon, w, z)|^{2} \leqq K(\epsilon, z, z) K(\epsilon, w, w)
$$

by the Schwarz inequality and since (7) holds by hypothesis, it follows from (50) that

$$
\left.\pi K(c, w, z)=\int_{0}^{c}(A(t, z), B(t, z)) d m(t) A(t, w), B(t, w)\right)^{-}
$$

for all complex $z$ and $w$. Therefore, if

$$
(f(t), g(t))=\chi(c, t)(A(t, w), B(t, w))
$$

the corresponding eigentransform is

$$
F(z)=K(c, \bar{w}, z) .
$$

Note that this function of $z$ is entire and that it does belong to $\mathfrak{H C}(E(c))$. Since $\operatorname{HC}(E(c))$ is contained isometrically in $L^{2}(\mu)$ by Theorem I,

$$
K(c, w, z)=\int K(c, w, t) \bar{K}(c, z, t) d \mu(t)
$$

for all complex $z$ and $w$. It follows from (56) and (58) that if $(f, g)$ is a finite linear combination of pairs of the form (57), where $w$ ranges in the complex plane, then the corresponding eigentransform $F(z)$ is an entire function, it belongs to $\mathfrak{H}(E(c))$, and (10) holds. Let $\mathfrak{T}(c)$ be the closure in $L_{0}^{2}(m)$ of such finite linear combinations of the form (57) with $w$ complex. Since $\mathfrak{T}(c)$ is a closed subspace of $L_{0}^{2}(m)$, the set of eigentransforms $F(z)$ of elements $(f, g)$ of $\mathfrak{M}(c)$ is a closed subspace of $\mathfrak{H}(E(c))$. We claim that it contains every element $G(z)$ of $\mathfrak{F C}(E(c))$. For let $F(z)$ be the projection of $G(z)$ into this set of eigentransforms. By construction, $K(c, w, z)$ is such an eigentransform for every complex number $w$. Therefore,

$$
G(w)=\langle G(t), K(c, w, t)\rangle=\langle F(t), K(c, w, t)\rangle=F(w) .
$$


By the arbitrariness of $w, G(z)=F(z)$ is the eigentransform of an element of $\mathfrak{M}(c)$. We will now show that $\mathfrak{T}(c)$ contains every element $(f, g)$ of $L_{0}^{2}(m)$ which is equivalent to zero outside of $[0, c]$. To do this, note that if $a$ is a regular point, and if $a<c$, and if $\mathfrak{T}(a)$ is defined accordingly, then for every $(f, g)$ in $\mathscr{T}(a)$, the corresponding eigentransform $F(z)$ belongs to $\mathfrak{H C}(E(a))$ and $(10)$ holds. Since $\mathfrak{H C}(E(a))$ is contained isometrically in $\mathfrak{F C}(E(c))$ by Theorem I, it follows that $(f, g)$ belongs to $\mathfrak{T}(c)$. In other words, $\mathfrak{T}(a)$ is contained in $\operatorname{Tr}(c)$. We claim that

$$
\chi(a, t)(f(t), g(t))
$$

belongs to $\mathfrak{T}(c)$ whenever $(f(t), g(t))$ belongs to $\mathscr{T}(c)$. This is obvious if $(f(t), g(t))$ is a finite linear combination of functions of the form (57), with $w$ complex, since then the product by $\chi(a, t)$ belongs to $\mathfrak{N}(a)$. The general case then follows by continuity. It follows by approximation that if $h(t)$ is any bounded measurable complex valued function, which remains constant on every interval of singular points, then

$$
h(t)(f(t), g(t))
$$

belongs to $\mathfrak{T}(c)$ whenever $(f(t), g(t))$ belongs to $\mathscr{T}(c)$. Since

$$
(\bar{A}(t, w), \bar{B}(t, w))=(A(t, \bar{w}), B(t, \bar{w})),
$$

we see similarly that $(\bar{f}(t), \bar{g}(t))$ belongs to $\mathscr{T}(c)$ whenever $(f(t), g(t))$ belongs to $\operatorname{Tl}(c)$. If $F(z)$ is the eigentransform of $(f, g)$, then $F^{*}(z)$ is the eigentransform of $(\bar{f}, \bar{g})$. This is obvious if $(f(t), g(t))$ is a finite linear combination of functions of the form (57). The general case follows by continuity. If $w$ is not real,

$$
(A(a, w), B(a, w)) \text { and }(\bar{A}(a, w), \bar{B}(a, w))
$$

are linearly independent since $E(a, z)$ satisfies (1). If $0<a<b \leqq c$, and if the interval $[a, b]$ contains only regular points, it follows from Lemma 5 that every pair $(f(t), g(t))$ of complex valued functions, which are continuous in $[a, b]$ and which vanish outside of $[a, b]$, belongs to $\mathscr{M}(c)$. Since $\operatorname{Tr}(c)$ is closed, it contains every element $(f(t), g(t))$ of $L_{0}^{2}(m)$ which is equivalent to zero outside of $[a, b]$. The same conclusion obviously holds if $(a, b)$ is an interval of singular points with $0 \leqq a<b \leqq c$, and $b$ regular, and $a$ regular or zero. It follows that $\mathfrak{T}(c)$ does contain every element of $L_{0}^{2}(m)$ which is equivalent to zero outside of $[0, c]$.

Proof of Theorem IIIB. Let $\left(f_{2}, g_{2}\right)$ be an element of $L_{0}^{2}(m)$ which is equivalent to zero outside of $[0, c]$, and let $F_{2}(z)$ be the corresponding eigentransform. Suppose that $F_{2}(0)=0$. Since $E(c, 0) \neq 0$ by hypothesis, $F_{1}(z)$ $=F_{2}(z) / z$ belongs to $\mathfrak{H}(E(c))$. By Theorem IIIA, there is a unique element $\left(f_{1}, g_{1}\right)$ of $L_{0}^{2}(m)$ which is equivalent to zero outside of $[0, c]$ and has $F_{1}(z)$ as its eigentransform. We will prove the theorem by showing that $\left(f_{1}, g_{1}\right)$ is 
in the domain of $H$ and that $\left(f_{2}, g_{2}\right)=H\left(f_{1}, g_{1}\right)$. To see this consider first a special case. Let $\alpha$ be a nonreal number, which will be held fixed. Let

$$
\begin{aligned}
L(c, w, z) & =\pi(z-\bar{w}) K(c, w, z) \\
& =B(c, z) \bar{A}(c, w)-A(c, z) \bar{B}(c, w) .
\end{aligned}
$$

Since

$$
L(c, \alpha, \alpha) L(c, w, z)=L(c, \bar{\alpha}, z) \bar{L}(c, \bar{\alpha}, w)-L(c, \alpha, z) \bar{L}(c, \alpha, w)
$$

for all complex $z$ and $w$, we have

$$
\begin{aligned}
& z(\alpha-\bar{\alpha}) K(c, \alpha, \alpha) K(c, w, z)-z(\bar{w}-\bar{\alpha}) \bar{K}(c, \bar{\alpha}, w) K(c, \bar{\alpha}, z) \\
& +z(\bar{w}-\alpha) \bar{K}(c, \alpha, w) K(c, \alpha, z)=\bar{w}(\alpha-\bar{\alpha}) K(c, \alpha, \alpha) K(c, w, z) \\
& \quad-\alpha(\bar{w}-\bar{\alpha}) \bar{K}(c, \bar{\alpha}, w) K(c, \bar{\alpha}, z)+\bar{\alpha}(\bar{w}-\alpha) \bar{K}(c, \alpha, w) K(c, \alpha, z) .
\end{aligned}
$$

If $w$ is held fixed and if

$$
\begin{aligned}
\left(f_{2}(t), g_{2}(t)\right)= & \bar{w}(\alpha-\bar{\alpha}) K(c, \alpha, \alpha) \chi(c, t)(A(t, \bar{w}), B(t, \bar{w})) \\
& -\alpha(\bar{w}-\bar{\alpha}) K(c, \bar{\alpha}, w) \chi(c, t)(A(t, \alpha), B(t, \alpha)) \\
& +\bar{\alpha}(\bar{w}-\alpha) K(c, \alpha, w) \chi(c, t)(A(t, \bar{\alpha}), B(t, \bar{\alpha})),
\end{aligned}
$$

then

$$
\begin{aligned}
F_{2}(z)= & \bar{w}(\alpha-\bar{\alpha}) K(c, \alpha, \alpha) K(c, w, z) \\
& -\alpha(\bar{w}-\bar{\alpha}) \bar{K}(c, \bar{\alpha}, w) K(c, \bar{\alpha}, z) \\
& +\bar{\alpha}(\bar{w}-\alpha) \bar{K}(c, \alpha, w) K(c, \alpha, z)
\end{aligned}
$$

for all complex $z$, and

$$
\begin{aligned}
F_{1}(z)= & (\alpha-\bar{\alpha}) K(c, \alpha, \alpha) K(c, w, z) \\
& -(\bar{w}-\alpha) \bar{K}(c, \bar{\alpha}, w) K(c, \bar{\alpha}, z) \\
& +(\bar{w}-\alpha) \bar{K}(c, \alpha, w) K(c, \alpha, z)
\end{aligned}
$$

and

$$
\begin{aligned}
\left(f_{1}(t), g_{1}(t)\right)= & (\alpha-\bar{\alpha}) K(c, \alpha, \alpha) \chi(c, t)(A(t, \bar{w}), B(t, \bar{w})) \\
& -(\bar{w}-\bar{\alpha}) \bar{K}(c, \bar{\alpha}, w) \chi(c, t)(A(t, \alpha), B(t, \alpha)) \\
& +(\bar{w}-\alpha) \bar{K}(c, \alpha, w) \chi(c, t)(A(t, \bar{\alpha}), B(t, \bar{\alpha})) .
\end{aligned}
$$

It follows from (59) that $f_{1}(c)=0$ and $g_{1}(c)=0$. Since $E(t, 0)=1$,

$$
B(t, z)=\pi z K(t, 0, z) .
$$

We saw in the proof of Theorem III that this quantity goes to zero with $t$ for each fixed $z$. Therefore, $g_{1}(0)=0$. It now follows from $(6)$ that $\left(f_{1}, g_{1}\right)$ is in the domain of $H$ and that $\left(f_{2}, g_{2}\right)=H\left(f_{1}, g_{1}\right)$. By linearity, the same conclusion holds if $\left(f_{2}, g_{2}\right)$ is a finite linear combination of functions of the form 
(60), where $w$ ranges in the complex plane. By continuity the same conclusion holds if $\left(f_{2}, g_{2}\right)$ belongs to the closure of such combinations. It remains to consider the case in which $\left(f_{2}, g_{2}\right)$ is orthogonal to all such combinations. By Theorem IIIA, $F_{2}(z)$ is then orthogonal to the right hand side of (61) for every complex number $w$. It follows that

$$
\begin{aligned}
0= & w(\bar{\alpha}-\alpha) K(c, \alpha, \alpha) F_{2}(w) \\
& -\bar{\alpha}(w-\alpha) K(c, \bar{\alpha}, w) F_{2}(\bar{\alpha}) \\
& +\alpha(w-\bar{\alpha}) K(c, \alpha, w) F_{2}(\alpha)
\end{aligned}
$$

for all complex $w$. Therefore, there exist complex numbers $u$ and $v$ such that

$$
z F_{2}(z)=A(c, z) u+B(c, z) v
$$

for all complex $z$. Since $E(c, 0)=1$, we must have $u=0$ and

$$
F_{2}(z)=\pi K(c, 0, z) v .
$$

Since $F_{2}(0)=0$, we must have $v=0$ and $F_{2}(z)$ vanishes identically. By Theorem IIIA, $\left(f_{1}, g_{1}\right)$ and $\left(f_{2}, g_{2}\right)$ vanish in this case. The theorem follows.

Proof of Theorem IIIC. If the set of regular points with respect to $m(t)$ is bounded, (4) implies that every element of $L_{0}^{2}(m)$ is equivalent to zero outside of $[0, c]$, where $c$ is the largest regular point. In this case, the theorem follows from Theorem IIIA and the fact that $\mathfrak{H C}(E(c))$ fills $L^{2}(\mu)$ by Theorem I. For the rest of the proof, we will suppose that the regular points are unbounded. Let $\left(c_{n}\right)$ be an increasing sequence of regular points which goes to infinity with $n$. Then,

$$
\left(f_{n}(t), g_{n}(t)\right)=\chi\left(c_{n}, t\right)(f(t), g(t))
$$

is in $L_{0}^{2}(m)$. Let $F_{n}(z)$ be the corresponding eigentransform. Since $(f, g)$ belongs to $L_{0}^{2}(m)$, the sequence $\left(\left(f_{n}, g_{n}\right)\right)$ is Cauchy in the metric of $L_{0}^{2}(m)$. By Theorem IIIA, $\left(F_{n}(z)\right)$ is a Cauchy sequence in the metric of $L^{2}(\mu)$. Since $L^{2}(\mu)$ is complete,

$$
F(x)=\lim F_{n}(x)
$$

converges in the metric of $L^{2}(\mu)$. Since (10) holds for every $\left(f_{n}, g_{n}\right)$, it holds for $(f, g)$. The reader should now have no difficulty verifying that (11) holds with convergence in the metric of $L^{2}(\mu)$. If $G(x)$ is in $L^{2}(\mu)$, let $F_{n}(z)$ be the projection of $G(x)$ in $\mathfrak{H C}\left(E\left(c_{n}\right)\right)$ for every $n$. By Theorem IIIA, $F_{n}(z)$ is the eigentransform of an element $\left(f_{n}(t), g_{n}(t)\right)$ of $L_{0}^{2}(m)$ which is equivalent to zero outside of $\left[0, c_{n}\right]$. Since $F_{m}(z)$ is the projection of $F_{n}(z)$ into $\mathcal{H}\left(E\left(c_{n}\right)\right)$ when $m \leqq n$,

$$
\left(f_{m}(t), g_{m}(t)\right)=\chi\left(c_{m}, t\right)\left(f_{n}(t), g_{n}(t)\right)
$$

Since 


$$
\begin{aligned}
\pi \int|F(t)|^{2} d \mu(t) & \geqq \pi \int\left|F_{n}(t)\right|^{2} d \mu(t) \\
& \geqq \int\left(f_{n}(t), g_{n}(t)\right) d m(t)\left(f_{n}(t), g_{n}(t)\right),
\end{aligned}
$$

there is an element $(f(t), g(t))$ of $L_{0}^{2}(m)$ such that

$$
\left(f_{n}(t), g_{n}(t)\right)=\chi\left(c_{n}, t\right)(f(t), g(t))
$$

for every $n$. Let $F(x)$ be its eigentransform. We have seen that

$$
F(x)=\lim F_{n}(x)
$$

with convergence in the metric of $L^{2}(\mu)$. By Theorem I,

$$
G(x)=\lim F_{n}(x)
$$

with convergence in the metric of $L^{2}(\mu)$. Therefore, $G(x)=F(x)$, a.e. with respect to $\mu$, is an eigentransform. Since $F_{n}^{*}(z)$ is the eigentransform of $\left(\bar{f}_{n}, \bar{g}_{n}\right)$ for every $n$ by Theorem IIIA, $\bar{F}_{n}(x)$ is the eigentransform of $(\bar{f}, \bar{g})$.

Proof of Theorem IIID, the sufficiency. If the regular points with respect to $m(t)$ are bounded, the theorem follows from Theorem I and Theorem IIIB. The necessity and sufficiency for Theorem IIID will be given an explicit proof only when the regular points are unbounded. By hypothesis, $\left(f_{1}, g_{1}\right)$ belongs to $L_{0}^{2}(m)$ and the corresponding eigentransform $F_{1}(x)$ has the property that $F_{2}(x)=x F_{1}(x)$ belongs to $L^{2}(\mu)$. By Theorem IIIC, $F_{2}(x)$ is the eigentransform of a unique element $\left(f_{2}, g_{2}\right)$ of $L_{0}^{2}(m)$. We must show that $\left(f_{1}, g_{1}\right)$ is in the domain of $H$ and that $\left(f_{2}, g_{2}\right)=H\left(f_{1}, g_{1}\right)$. For each regular $c>0$, let $F_{i}(c, z)$ be the projection of $F_{i}(x)$ into $\mathfrak{H C}(E(c)), i=1,2$. Then,

$$
\left[F_{2}(c, z)-A(c, z) F_{2}(c, 0)\right] / z
$$

belongs to $\mathfrak{H C}(E(c))$. Let $G(z)$ be any element of $\mathfrak{H C}(E(c))$ such that $G(0)=0$. Then,

$$
\begin{aligned}
\left\langle\left[F_{2}(c, t)-A(c, t) F_{2}(c, 0)\right] / t, G(t)\right\rangle & =\left\langle F_{2}(c, t), G(t) / t\right\rangle \\
& =\left\langle F_{2}(t), G(t) / t\right\rangle \\
& =\left\langle F_{1}(t), G(t)\right\rangle \\
& =\left\langle F_{1}(c, t), G(t)\right\rangle
\end{aligned}
$$

since $G(z) / z$ belongs to $\Re(E(c))$. By the arbitrariness of $G(z)$, there is a unique complex number $u(c)$ such that $\left[F_{2}(c, z)-A(c, z) F_{2}(c, 0)\right] / z+K(c, 0, z) u(c)$ $=F_{1}(c, z)$ for all complex $z$. Let

$$
v(c)=\pi F_{2}(c, 0)
$$


Then,

$$
\pi F_{2}(c, z)=\pi z F_{1}(c, z)+A(c, z) v(c)-B(c, z) u(c)
$$

for all complex $z$. Let $\alpha$ be a nonreal number. Then,

$$
\begin{aligned}
\pi(\alpha- & \bar{\alpha}) K(c, \alpha, \alpha) z F_{1}(c, z) \\
& +[u(c) B(c, \bar{\alpha})-v(c) A(c, \bar{\alpha})] z K(\bar{\alpha}, z) \\
& -[u(c) B(c, \alpha)-v(c) A(c, \alpha)] z K(\alpha, z) \\
= & \pi(\alpha-\bar{\alpha}) K(c, \alpha, \alpha) F_{2}(c, z) \\
& +[u(c) B(c, \bar{\alpha})-v(c) A(c, \bar{\alpha})] \alpha K(\bar{\alpha}, z) \\
& -[u(c) B(c, \alpha)-v(c) A(c, \alpha)] \bar{\alpha} K(\alpha, z)
\end{aligned}
$$

for all complex z. By Theorem IIIB,

$$
\begin{aligned}
\pi(\alpha & -\bar{\alpha}) K(c, \alpha, \alpha) \chi(c, t)\left(f_{1}(t), g_{1}(t)\right) \\
+ & {[u(c) B(c, \bar{\alpha})-v(c) A(c, \bar{\alpha})] \chi(c, t)(A(t, \alpha), B(t, \alpha)) } \\
- & {[u(c) B(c, \alpha)-v(c) A(c, \alpha)] \chi(c, t)(A(t, \bar{\alpha}), B(t, \bar{\alpha})) }
\end{aligned}
$$

is in the domain of $H$ and the action of $H$ yields

$$
\begin{aligned}
\pi(\alpha- & \bar{\alpha}) K(c, \alpha, \alpha) \chi(c, t)\left(f_{2}(t), g_{2}(t)\right) \\
+ & {[u(c) B(c, \bar{\alpha})-v(c) A(c, \bar{\alpha})] \alpha \chi(c, t)(A(t, \alpha), B(t, \alpha)) } \\
- & {[u(c) B(c, \alpha)-v(c) A(c, \alpha)] \bar{\alpha} \chi(c, t)(A(t, \bar{\alpha}), B(t, \bar{\alpha})) . }
\end{aligned}
$$

By (6) and the arbitrariness of $c,\left(f_{1}, g_{1}\right)$ is in the domain of $H$ and $\left(f_{2}, g_{2}\right)$ $=H\left(f_{1}, g_{1}\right)$. Incidentally, the definition of $H$ implies that

$$
u(c)=f_{1}(c) \quad \text { and } \quad v(c)=g_{1}(c) .
$$

Proof of Theorem IIID, the necessity. The hypothesis is that $\left(f_{1}, g_{1}\right)$ is in the domain of $H$ and that $\left(f_{2}, g_{2}\right)=H\left(f_{1}, g_{1}\right)$. Let $F_{i}(c, z)$ be defined as in the proof of sufficiency, $i=1,2$, when $c$ is regular with respect to $m(t)$. By reversing the arguments in the proof of sufficiency, we find that

$$
\pi F_{2}(c, z)=\pi z F_{1}(c, z)+A(c, z) g_{1}(c)-B(c, z) f_{1}(c)
$$

for all complex $z$. Let $w$ be a nonreal number, which will be held fixed, and let

$$
G(x)=\pi F_{1}(x)-\pi\left[F_{2}(x)-w F_{1}(x)\right] /(x-w) .
$$

We will prove the necessity by showing that $G(x)$ vanishes a.e. with respect to $\mu$. Certainly, $G(x)$ belongs to $L^{2}(\mu)$. Let $G(c, z)$ be the projection of $G(x)$ into $\mathfrak{H C}(E(c))$ when $c$ is regular. Let $L(z)$ be any element of $\mathfrak{H}(E(c))$ such that $z L(z)$ belongs to $\mathfrak{H C}(E(c))$. Since $(z-\bar{w}) L(z)$ is in $\mathfrak{H}(E(c))$, 


$$
\begin{aligned}
\langle G(c, t),(t-\bar{w}) L(t)\rangle= & \langle G(t),(t-\bar{w}) L(t)\rangle \\
= & \pi\left\langle F_{1}(t),(t-\bar{w}) L(t)\right\rangle \\
& -\pi\left\langle\left[F_{2}(t)-w F_{1}(t)\right] /(t-w),(t-\bar{w}) L(t)\right\rangle \\
= & \pi\left\langle F_{1}(t),(t-\bar{w}) L(t)\right\rangle \\
& -\pi\left\langle F_{2}(t)-w F_{1}(t), L(t)\right\rangle \\
= & \pi\left\langle F_{1}(c, t),(t-\bar{w}) L(t)\right\rangle \\
& -\pi\left\langle F_{2}(c, t)-w F_{1}(c, t), L(t)\right\rangle=0
\end{aligned}
$$

by (62). By the arbitrariness of $L(z)$, there is some complex number $u(c)$ such that

$$
G(c, z)=K(\bar{w}, z) u(c)
$$

for all complex $z$. Since $G(a, z)$ is the projection of $G(b, z)$ into $\mathfrak{H C}(E(a))$ when $a$ and $b$ are regular and $a \leqq b, u(a)=u(b)=u$ is independent of $a$ and $b$. Therefore,

$$
G(c, z)=K(\bar{w}, z) u
$$

and

$$
\|G(t)\|^{2} \geqq\|G(c, t)\|^{2}=K(c, \bar{w}, \bar{w})|u|^{2} .
$$

By (8) and the arbitrariness of $c, u=0$. It follows that $G(c, z)=0$ for all complex $z$ when $c$ is regular, and that $G(x)=\lim G(c, x)$ vanishes a.e. with respect to $\mu$. The necessity follows.

Proof of Theorem IV, the sufficiency. The hypothesis (12) implies that

$$
z \bar{w} K(w+1, z+1)=K(w, z)-\pi \alpha^{-1} K(0, z) K(w, 0)
$$

for all complex $z$ and $w$ since $\operatorname{det} P=1$. It follows that if

$$
F(z)=\bar{w} K(w+1, z)
$$

for some complex number $w$, then

$$
z F(z+1)=z \bar{w} K(w+1, z+1)
$$

is in $\mathfrak{H C}(E)$. Furthermore, for any complex numbers $w_{1}$ and $w_{2}$,

$$
\begin{gathered}
\left\langle t \bar{w}_{1} K\left(w_{1}+1, t+1\right), t \bar{w}_{2} K\left(w_{2}+1, t+1\right)\right\rangle \\
=\left\langle\bar{w}_{1} K\left(w_{1}+1, t\right), \bar{w}_{2} K\left(w_{2}+1, t\right)\right\rangle
\end{gathered}
$$

as a result of (63). By linearity, it follows that if $F(z)$ is any finite linear combination of functions of the form $\bar{w} K(w+1, z)$, with $w$ complex, then $z F(z+1)$ belongs to $\mathcal{H}(E)$ and has the same norm as $F(z)$. Since such combinations are dense in $\mathfrak{H C}(E)$, we see by continuity that $\mathfrak{H C}(E)$ satisfies (I). To see that $\mathfrak{H C}(E)$ satisfies (II), we must show that if $G(z)$ is any element of $\mathfrak{H C}(E)$ orthog- 
onal to every $z F(z+1)$, with $F(z)$ in $\mathfrak{H C}(E)$, then $G(z)$ is a constant multiple of $K(0, z)$. In this case, $G(z)$ is orthogonal to the left hand side, and hence also to the right hand side, of (63) for every complex number $w$. It follows that

$$
G(w)=\pi \alpha^{-1} G(0) K(0, w)
$$

and that $G(z)$ is a constant multiple of $K(0, z)$.

Proof of Theorem IV, the necessity. We establish (63) by showing that left and right hand sides have the same inner product with every element $G(z)$ of $\mathfrak{H C}(E)$. This is clear if $G(z)$ is a constant multiple of $K(0, z)$. Therefore, it is sufficient to consider the case that $G(0)=0$. By (II), we then have $G(z)$ $=z F(z+1)$ for some $F(z)$ in $\mathfrak{F C}(E)$. Because of $(\mathrm{I})$,

$$
\begin{aligned}
& \langle t F(t+1), t \bar{w} K(w+1, t+1)\rangle \\
& \quad=\langle F(t), \bar{w} K(w+1, t)\rangle=w F(w+1) \\
& \quad=\left\langle t F(t+1), K(w, t)-\pi \alpha^{-1} K(0, t) K(w, 0)\right\rangle
\end{aligned}
$$

as desired. Formula (63) now follows. The reader should be able to deduce from (63) that (12) holds for some matrix $P$ of real numbers such that $\operatorname{det} P=1$.

Proof of Theorem V. The reader should have no difficulty verifying that the integral in the definition of $v(t)$ is absolutely convergent and that (21) holds when $0<a<1$. To see that the integral in the definition of $\beta(t)$ is absolutely convergent, we must verify that

$$
\int_{0}^{a} \int_{t}^{1}\left(s \log s^{-1}\right)^{-2} d s d t
$$

is finite when $0<a<1$. Since

$$
\int_{t}^{1}\left(s \log s^{-1}\right)^{-2} d s=o\left(t^{-1}\right)
$$

as $t \searrow 0$, the convergence of (64) becomes equivalent to that of

$$
\int_{0}^{a} t^{-1}\left(\log t^{-1}\right)^{-2} d t=\left(\log a^{-1}\right)^{-1}
$$

on integration by parts. Therefore, $\beta(t)$ is well-defined. It is now easily seen that $\beta^{\prime}(t)=v(t)$ for $0<t<1$. The choice of constant in the definition of $\gamma(t)$ does not matter for our construction. The function $m(t)$ is well-defined for $0<t<1$ and both (2) and (3) are satisfied. The finiteness of $\gamma(1)$ follows from our definition of $v(t)$ and the fact that

$$
\int_{a}^{1}\left(t \log \log t^{-1}\right)^{-2} d t<\infty
$$


for $0<a<1$. The finiteness of $\beta(1)$ now follows by the Schwarz inequality since

$$
\beta^{\prime}(t)^{2}=\alpha^{\prime}(t) \gamma^{\prime}(t)
$$

where $\alpha(1)$ and $\gamma(1)$ are finite. To see that (14) is satisfied, we must verify that $t v^{2}(t)$ is integrable in a neighborhood of the origin. This follows from the fact that

$$
v(t) \sim(t \log t)^{-1}
$$

as $t \searrow 0$, which is true because

$$
\begin{aligned}
\frac{d}{d t}(t \log t)^{-1} & =\frac{1}{t^{2} \log t^{-1}}\left(1-\frac{1}{\log t^{-1}}\right) \\
& \sim v^{\prime}(t)
\end{aligned}
$$

as $t \searrow 0$. The existence of the desired family $(E(t, z))$ satisfying (6) and (15) now follows from Theorem IV of [10]. We have yet to show that (13) holds. To see this, substitute the expression (19) for $P(t)$ in (18) and verify that the equation is indeed satisfied because of (20) and (21). Since $P(t)$ so defined commutes with $m^{\prime}(t) I$, it is now easily verified that (17) holds for all complex $z$ when $0<t<1$. Now we will show that (16) holds. To see this define

$$
\begin{aligned}
(F(t, z), G(t, z))= & z(A(t, z+1), B(t, z+1)) P(t) \\
& -(A(t, z), B(t, z))\left(\begin{array}{cc}
1 & 0 \\
-z^{-1} \alpha(t)^{-1} & 1
\end{array}\right) .
\end{aligned}
$$

Now (6) and (17) imply that

$$
\begin{aligned}
\frac{\partial}{\partial t}(F(t, z), G(t, z))= & -(z+1)(F(t, z), G(t, z)) m^{\prime}(t) I \\
& +(F(t, z), G(t, z)) P(t)^{-1} P^{\prime}(t)
\end{aligned}
$$

The most general solution of this differential equation is of the form

$$
\begin{aligned}
& F(t, z)=R(z) z A(t, z+1) p(t)+R(z) z B(t, z+1) r(t), \\
& G(t, z)=T(z) z A(t, z+1) q(t)+T(z) z B(t, z+1) s(t),
\end{aligned}
$$

where $R(z)$ and $T(z)$ are complex valued functions of $z$. Therefore,

$$
\begin{aligned}
& {[1-R(z)] z A(t, z+1) p(t)+[1-R(z)] z B(t, z+1) r(t)=A(t, z)-z^{-1} t^{-1} B(t, z),} \\
& {[1-T(z)] z A(t, z+1) q(t)+[1-T(z)] z B(t, z+1) s(t)=B(t, z)}
\end{aligned}
$$

for all complex $z$ when $0 \leqq t \leqq 1$. If we multiply each side of the second equation by $\exp [\beta(t) z]$, substitute from (19), and pass to a limit using (15), we find that 


$$
1-T(z)=\lim _{t \searrow 0}\left(\log t^{-1}\right)^{-1} \exp \beta(t) .
$$

But our definition of $\beta(t)$ is made so that the limit on the right is 1 . Therefore, $T(z)$ vanishes identically. A similar argument shows that $R(z)$ vanishes identically. Formula (16) follows. Now observe that

$$
\lim _{t / 1} P(t)=1 \text {. }
$$

This follows from (19) and the definition of $v(t)$ since

$$
\begin{array}{ll}
\lim _{t / 1} \log t & =0, \\
\lim _{t / 1} \log t \log \log t^{-1} & =0, \\
\lim _{t / 1} \log t\left(\log \log t^{-1}\right)^{2} & =0 .
\end{array}
$$

Therefore, $A(1, z)$ and $B(1, z)$ satisfy $(12)$ with $P=1$ and $\alpha=1$. Before completing the proof of Theorem $\mathrm{V}$, we interrupt briefly for the proof of Theorem VI, which can help us complete the present proof.

Proof of Theorem VI. Because of (20), the statement that $\phi(t, w)$ belongs to $L^{2}(0,1)$ is equivalent to the statement that

$$
\chi(1, t)(A(t, w), B(t, w))
$$

belongs to $L_{0}^{2}(m)$, which is true because of Theorem IIIA. The rest of Theorem VIA follows similarly from Theorem IIIA using the natural correspondence between $L_{0}^{2}(m)$ and $L^{2}(0,1)$. Theorem VIB is a similar translation of Theorem IIIB, and the reader can probably make it as rapidly by himself as we could do it for him.

Proof of Theorem V, continued. We have seen that $A(1, z)$ and $B(1, z)$ satisfy (12) with $P=1$ and $\alpha=1$. In particular, $B(1, z)$ is an entire function such that

$$
z B(1, z+1)=B(1, z)
$$

for all complex $z$. Therefore, $B(1, z)$ has zeros at $0,-1,-2, \cdots$, and $B(1, z) \Gamma(z)$ is a periodic entire function of period 1 . The zeros of $B(1, z)$ are known to be real because of (1). We will show that $B(1, z) \Gamma(z)$ has no zeros. By periodicity, we need only show that $B(1, z)$ has no positive zeros. In fact, if $w$ is a zero of $B(1, z)$, the quotient $B(1, z) /(z-w)$ is in $\mathcal{F}(E(1))$ since $E(1, z)$ has no real zeros by Theorem IV of $[10]$. But then $B(1, z) /(z-w)$ is a constant multiple of $K(1, w, z)$, which is the eigentransform of $\phi(t, w)$, in the notation of Theorem VIA. If we pass to the situation of Theorem VIB, formula (6) implies that $\phi(t, w)$ is an absolutely continuous function of $t$ such that 


$$
v^{\prime}(a)^{-1} \phi^{\prime}(a, w)=w \int_{0}^{a} \phi(t, w) d t
$$

for $0<a<1$. But if $w \neq 0, B(1, z) /(z-w)$ vanishes at the origin. It follows that $K(1, w, z)$ is orthogonal in $\nVdash(E(1))$ to $K(1,0, z)$, which is the eigentransform of the constant function 1 in $L^{2}(0,1)$. Formula (10) now implies that

$$
\int_{0}^{1} \phi(t, w) d t=0
$$

Therefore, we may integrate by parts to find that

$$
w \int_{0}^{1} \phi(t, w)^{2} d t=-\int_{0}^{1} \phi^{\prime}(t, w)^{2} v^{\prime}(t)^{-1} d t .
$$

Since $v^{\prime}(t) \geqq 0$ by construction and since $\phi(t, w)$ is a real valued function of $t$ when $w$ is real, we conclude that $w \leqq 0$. This completes the proof that $B(1, z)$ has no positive zeros, and hence establishes that $B(1, z) \Gamma(z)$ has no zeros. Because of Theorem IV of $[10], B(1, z)$ is an entire function of genus 0 or 1 . Since $S(z)$ has genus 1 , the function $B(1, z) \Gamma(z)$ has genus 0 or 1 . Since it has no zeros, it is the product of a constant and an exponential. Since $B(1, z)$ is real for real $z$ by construction and since $\Gamma(z)$ is real for real $z$ by its factorization, $B(1, z) \Gamma(z)$ is real for real $z$. Since this function is also known to be periodic of period 1 , it is a constant. But $B(1, z) / z=\alpha(1)=1$ when $z=0$ by construction and $S(z) / z=1$ when $z=0$ by the infinite product. Therefore, $B(1, z) \Gamma(z)=1$ identically and $B(1, z)=S(z)$. Since $A(1, z)$ and $B(1, z)$ satisfy (12),

$$
z A(1, z+1)=A(1, z)-S(z) / z
$$

for all complex $z$. The identity

$$
z S^{\prime}(z+1)=S^{\prime}(z)-S(z) / z
$$

follows from the identity $z S(z+1)=S(z)$ on differentiating. Therefore,

$$
z\left[A(1, z+1)-S^{\prime}(z+1)\right]=A(1, z)-S^{\prime}(z)
$$

for all complex $z$. It follows that the entire function $A(1, z)-S^{\prime}(z)$ vanishes at the nonpositive integers and that

$$
U(z)=\left[A(1, z)-S^{\prime}(z)\right] \Gamma(z)
$$

is a periodic entire function of period 1 . We may also write

$$
U(z)=A(1, z) / B(1, z)-S^{\prime}(z) / S(z)
$$

where 


$$
\begin{aligned}
\operatorname{Re} i A(1, z) / B(1, z) & >0, \\
\operatorname{Re} i S^{\prime}(z) / S(z) & >0
\end{aligned}
$$

for $y>0$ because $A(1, z)-i B(1, z)$ and $S^{\prime}(z)-i S(z)$ satisfy (1). By the Poisson representation of a function positive and harmonic in a half-plane,

$$
\operatorname{Re} i U(z)=\frac{y}{\pi} \int \frac{\operatorname{Re} i U(t) d t}{(t-x)^{2}+y^{2}}+k y
$$

for $y>0$, with absolute convergence of the integral, where $k$ is a real constant. Since $U(z)$ is real for real $z$ by construction, the integral may be omitted in this formula. Since there are no singular points for $m(t), B(1, z)$ does not belong to $\mathfrak{H C}(E(1))$ and

$$
A(1, i y) / B(1, i y)=o(y)
$$

as $y \rightarrow+\infty$. By the infinite product for $S(z)$,

$$
S^{\prime}(i y) / S(i y)=o(y)
$$

as $y \rightarrow+\infty$. It now follows that $k=0$. We have shown that $\operatorname{Re} i U(z)=0$ for $y>0$. By the Cauchy-Riemann equations, $U(z)=U$ is a constant for $y>0$. By analytic continuation, the same is true for all complex $z$. We have

$$
A(1, z)=S^{\prime}(z)+U S(z)
$$

for all complex $z$. On computing derivatives at the origin, we find that

$$
-\beta(1)=S^{\prime \prime}(0)+U S^{\prime}(0) \text {. }
$$

To determine $\beta(1)$, we must use the formula

$$
\beta(t)=t v(t)+\log \log t^{-1} .
$$

To see that it is valid, note that the derivative of the right hand side of the proposed identity is $v(t)$. Therefore, left and right hand sides can differ only by a constant. This constant is zero since

$$
\lim _{t \searrow 0}\left[\beta(t)-\log \log t^{-1}\right]=0
$$

by definition and

$$
\lim _{t \supset 0}[t v(t)]=0
$$

since

$$
v(t) \sim(t \log t)^{-1}
$$

as we have seen. Therefore,

$$
\beta(1)=\lim _{t / 1}\left[t v(t)+\log \log t^{-1}\right]=-S^{\prime \prime}(0)
$$


by our definition of $v(t)$. It follows that

$$
S^{\prime \prime}(0)=S^{\prime \prime}(0)+U S^{\prime}(0)
$$

and that $U=0$ since $S^{\prime}(0)=1$. This completes the proof of the theorem.

Proof of Theorem VII. We have seen that $\phi(t, w)$ belongs to $L^{2}(0,1)$ as a function of $t$ for every complex number $w$. Since (6) and (16) hold, we have the identity

$$
\bar{w} \phi(t, \bar{w}+1)=\phi(t, \bar{w})-t^{-1} \int_{0}^{t} \phi(s, \bar{w}) d s
$$

for every complex number $w$. Therefore, if

$$
f(t)=\phi(t, \bar{w})-\int_{0}^{1} \phi(s, \bar{w}) d s
$$

and if $g(t)$ is defined by (22), then

$$
g(t)=\bar{w} \phi(t, \bar{w}+1) .
$$

The corresponding eigentransforms are

$$
\begin{aligned}
& F(z)=K(1, w, z)-\pi K(1,0, z) K(1, w, 0), \\
& G(z)=\bar{w} K(1, w+1, z) .
\end{aligned}
$$

The identity $F(z)=z G(z+1)$ is now (63). The theorem follows by linearity and continuity. It should be mentioned that the functions $\phi(t, w)$, where $w$ ranges in the nonpositive integers, form an orthogonal basis for $L^{2}(0,1)$ as a result of Theorems $\mathrm{V}$ and VIA, since the corresponding eigentransforms $K(w, z)$ form an orthogonal basis for $\mathfrak{H C}(E(1))$.

Proof of Theorem VIII. If $F(x)$ is a finite linear combination of the functions $(x-w)^{-1}$, where $w$ is not real, the existence of $G(x)$ defined by (31), and formula (32), follow from formula (30). The general case then follows by linearity and continuity. The only other information necessary for existence and uniqueness is that the finite linear combinations of the functions, $(x-w)^{-1}$, where $w$ is not real, are dense in $L^{2}(\mu)$ and in $L^{2}(\nu)$. This follows from properties of the Poisson kernel and should need no proof here. Similar considerations are necessary for the development of the ordinary Hilbert transform by Titchmarsh [14, Chapter V].

Proof of Theorem IX. By the proof of sufficiency for Theorem IV of [8],

$$
\frac{A(z)-A(w)}{z-w} \text { and } \frac{B(z)-B(w)}{z-w}
$$

belong to $\mathfrak{H C}(A-i B)$ as functions of $z$ for every complex number $w$. Since

$$
L(w, z)=\frac{\bar{C}(w)}{\pi} \frac{B(z)-B(\bar{w})}{z-\bar{w}}-\frac{\bar{D}(w)}{\pi} \frac{A(z)-A(\bar{w})}{z-\bar{w}},
$$


$L(w, z)$ belongs to $\mathfrak{H}(A-i B)$ as a function of $z$ for every complex number $w$. If we take $F(z)=K\left(w_{1}, z\right)$ in (35) for some fixed complex number $w_{1}$, we find that

$$
G(w)=\left\langle L(w, t), K\left(w_{1}, t\right)\right\rangle_{A-i B}^{-}=L\left(w, w_{1}\right)^{-}
$$

so that

$$
G(z)=\frac{1-D(z) \bar{A}\left(w_{1}\right)+C(z) \bar{B}\left(w_{1}\right)}{\pi\left(\bar{w}_{1}-z\right)} .
$$

With a change of variable, the proof of sufficiency for Theorem IV of [8] shows that

$$
\frac{C(z)-C(w)}{z-w} \text { and } \frac{D(z)-D(w)}{z-w}
$$

belong to $\mathfrak{F}(D+i C)$ as functions of $z$ for every complex number $w$. By taking a linear combination, we find that the function $G(z)$ just obtained does belong to $\operatorname{IC}(D+i C)$ as a function of $z$. To prove the theorem, we must verify that for all complex numbers $z$ and $w$,

$$
\begin{array}{r}
\left\langle\frac{1-D(t) \bar{A}(w)+C(t) \bar{B}(w)}{\pi(\bar{w}-t)}, \frac{1-D(t) \bar{A}(z)+C(t) \bar{B}(z)}{\pi(\bar{z}-t)}\right\rangle_{D+i C} \\
=\frac{B(z) \bar{A}(w)-A(z) \bar{B}(w)}{\pi(z-w \bar{w})}
\end{array}
$$

since this last expression is equal to

$$
\langle K(w, t), K(z, t)\rangle_{A-i B} .
$$

The theorem will then follow by linearity and continuity. Formula (65) is a consequence of the four formulas

$$
\begin{aligned}
& \left\langle\frac{D(t)-D(z)}{t-z}, \frac{D(t)-D(w)}{t-w}\right\rangle_{D+i C}=\pi \frac{\bar{D}(w) B(z)-\bar{B}(w) D(z)}{z-\bar{w}} \\
& \left\langle\frac{D(t)-D(z)}{t-z}, \frac{C(t)-C(w)}{t-w}\right\rangle_{D+i C}=\pi \frac{1-\bar{A}(w) D(z)+\bar{C}(w) B(z)}{z-\bar{w}} \\
& \left\langle\frac{C(t)-C(z)}{t-z}, \frac{D(t)-D(w)}{t-w}\right\rangle_{D+i C}=-\pi \frac{1-\bar{D}(w) A(z)+\bar{B}(w) C(z)}{z-\bar{w}}, \\
& \left\langle\frac{C(t)-C(z)}{t-z}, \frac{C(t)-C(w)}{t-w}\right\rangle_{D+i C}=\pi \frac{\bar{C}(w) A(z)-\bar{A}(w) C(z)}{z-\bar{w}} .
\end{aligned}
$$

The proofs of formulas (66), (67), (68), and (69) are closely analogous to the proof of formula (21) of [8], and it should be unnecessary to go through this 
process again here. The required partial fraction decompositions are possible because of (34).

Proof of Theorem $\mathbf{X}$. The isometric inclusions of $\mathfrak{F C}(A-i B)$ in $L^{2}(\mu)$ and of $\mathfrak{F C}(D+i C)$ in $L^{2}(\nu)$ follow by Theorem $\mathrm{V}$ of [8] because of (37) and (39). Formulas (26) and (28) then follow by the proof of Theorem VI of [8]. It remains to show that (31) holds when $F(z)$ and $G(z)$ are related by (35). For this, we will need to compute the $L^{2}(\mu)$ projection $P(w, z)$ of $(x-\bar{w})^{-1}$ into $\mathcal{H}(A-i B)$ when $w$ is not real and the $L^{2}(\nu)$ projection $Q(w, z)$ of $(x-\bar{w})^{-1}$ into $\mathfrak{F}(D+i C)$. These are given by the integrals

$$
\begin{aligned}
& P(w, z)=\int \frac{B(t) A(z)-A(t) B(z)}{\pi(t-z)} \frac{d \mu(t)}{(t-\bar{w})}, \\
& Q(w, z)=\int \frac{D(t) C(z)-C(t) D(z)}{\pi(t-z)} \frac{d \nu(t)}{(t-\bar{w})} .
\end{aligned}
$$

To evaluate then, we use formulas (27) and (28) of [8] in the form

$$
\begin{aligned}
\frac{i(\bar{w}-z)}{\pi} \int \frac{A(t)+i B(t)}{(t-z)(t-\bar{w})} d \mu(t) & =\frac{2}{[A(z)-i B(z)]-[A(z)+i B(z)] W(z)} \\
& +\frac{2 \bar{W}(w)}{[A(\bar{w})+i B(\bar{w})]-[A(\bar{w})-i B(\bar{w})] \bar{W}(w)}, \\
\frac{i(\bar{w}-z)}{\pi} \int \frac{A(t)-i B(t)}{(t-z)(t-\bar{w})} d \mu(t) & =\frac{2 W(z)}{[A(z)-i B(z)]-[A(z)+i B(z)] W(z)} \\
& +\frac{2}{[A(\bar{w})+i B(\bar{w})]-[A(\bar{w})-i B(\bar{w})] \bar{W}(w)}
\end{aligned}
$$

when $i(\bar{z}-z)>0$ and $i(\bar{w}-w)>0$. It follows that

$$
\begin{aligned}
P(w, z)= & -\frac{A(z)-A(\bar{w})}{z-\bar{w}} \frac{1-\bar{W}(w)}{[A(\bar{w})+i B(\bar{w})]-[A(\bar{w})-i B(\bar{w})] \bar{W}(w)} \\
& -2 i \frac{B(z)-B(\bar{w})}{z-\bar{w}} \frac{1+\bar{W}(w)}{[A(\bar{w})+i B(\bar{w})]-[A(\bar{w})-i B(\bar{w})] \bar{W}(w)}
\end{aligned}
$$

when $i(\bar{w}-w)>0$. A similar computation in $\mathfrak{C}(D+i C)$ yields

$$
\begin{aligned}
Q(w, z)= & -\frac{C(z)-C(\bar{w})}{z-\bar{w}} \frac{1-\bar{W}(w)}{[C(\bar{w})+i D(\bar{w})]-[C(\bar{w})-i D(\bar{w})] \bar{W}(w)} \\
& -2 i \frac{D(z)-D(\bar{w})}{z-\bar{w}} \frac{1+\bar{W}(w)}{[C(\bar{w})+i D(\bar{w})]-[C(\bar{w})-i D(\bar{w})] \bar{W}(w)}
\end{aligned}
$$

when $i(\bar{w}-w)>0$. We now use the formulas to (66), (67), (68), and (69) in $\mathcal{H C}(A-i B)$ : 


$$
\begin{aligned}
& \left\langle\frac{A(t)-A(z)}{t-z}, \frac{A(t)-A(w)}{t-w}\right\rangle_{A-i B}=\pi \frac{\bar{C}(w) A(z)-\bar{A}(w) C(z)}{z-w}, \\
& \left\langle\frac{A(t)-A(z)}{t-z}, \frac{B(t)-B(w)}{t-w}\right\rangle_{A-i B}=-\pi \frac{1-\bar{D}(w) A(z)+\bar{B}(w) C(z)}{z-\bar{w}}, \\
& \left\langle\frac{B(t)-B(z)}{t-z}, \frac{A(t)-A(w)}{t-w}\right\rangle_{A-i B}=\pi \frac{1-\bar{A}(w) D(z)+\bar{C}(w) B(z)}{z-\bar{w}}, \\
& \left\langle\frac{B(t)-B(z)}{t-z}, \frac{B(t)-B(w)}{t-w}\right\rangle_{A-i B}=\pi \frac{\bar{D}(w) B(z)-\bar{B}(w) D(z)}{z-\bar{w}} .
\end{aligned}
$$

It follows from (70) using (72), (73), (74), and (75) that

$$
\langle P(w, t), L(z, t)\rangle_{A-i B}=i \Phi(w) Q(w, z)
$$

when $i(\bar{w}-w)>0$. A similar analysis in the lower half-plane will show that (76) holds whenever $w$ is not real. Now return to functions $F(z)$ and $G(z)$, as in the statement of the theorem, which are related by (35). We wish to show that (31) holds for every nonreal number $w$. Since $F(z)$ is in $\mathfrak{F C}(A-i B)$ and $G(z)$ is in $\mathfrak{F C}(D+i C)$ by hypothesis, it is sufficient to show that

$$
\langle G(t), Q(w, t)\rangle_{D+i C}=i \phi(w)^{-1}\langle F(t), P(w, t)\rangle_{A-i B} .
$$

This formula follows from (76) and the isometric character of the transformation defined by (35).

Proof of Theorem XI. The first formula we are required to establish follows from the identity

$$
L^{*}(w, z)=L(\bar{w}, z)
$$

which holds for each fixed complex number $w$; the star is taken of $L(w, z)$ as a function of $z$. The second formula is most easily established in special cases. The most general case then follows by linearity and continuity. Let $w$ in the statement of the theorem be replaced by $w_{0}$, which is held fixed. It is sufficient to consider cases in which $F(z)$ is

$$
\frac{A(z)-A(\bar{w})}{z-\bar{w}} \text { or } \frac{B(z)-B(\bar{w})}{z-\bar{w}}
$$

for some number $w \neq w_{0}$. The corresponding transform, defined by (35), is

$$
\frac{C(z)-C(\bar{w})}{z-\bar{w}} \text { or } \frac{D(z)-D(\bar{w})}{z-\bar{w}},
$$

respectively, because of formulas (72), (73), (74), and (75). The reader will have no difficulty verifying that the formula of the theorem is correct in these special cases.

Proof of Theorem XIIA. This part of the theorem follows from formula (10) of Theorem IIIA once we show that 


$$
L(c, w, z)=\int_{0}^{c}(C(t, w), D(t, w)) d m(t)(A(t, \bar{z}), B(t, \bar{z}))^{-}
$$

for all complex $z$ and $w$. To see this, note that since $C(t, \bar{w})$ and $D(t, \bar{w})$ are continuous functions of $t \geqq 0$ for every complex number $w$ and since (40) holds,

$$
\int_{0}^{c}(C(t, \bar{w}), D(t, \bar{w})) d m(t)(C(t, \bar{w}), D(t, \bar{w}))^{-}<\infty
$$

Therefore, the integral in (77) is absolutely convergent for every $z$ and $w$. As in the proof of formula (50), it is sufficient to establish (77) when $z \neq \bar{w}$. The formula now follows from (41). The reader will have no trouble making the derivation if he will write both formulas in differential form.

Proof of Theorem XIIB. This part of the theorem follows from Part A using Theorem IIIC. Of course, formula (46) is just formula (11) with a change of variables.

\section{REFERENCES}

1. R. P. Boas, Jr., Entire functions, New York, Academic Press, 1954.

2. L. de Branges, Local operators on Fourier transforms, Duke Math. J. vol. 25 (1958) pp. $143-154$.

3. — The a-local operator problem, Canad. J. Math. vol. 11 (1959) pp. 583-592.

4. - The Stone-Weierstrass theorem, Proc. Amer. Math. Soc. vol. 10 (1959) pp. 822824.

5. - The Bernstein problem, Proc. Amer. Math. Soc. vol. 10 (1959) pp. 825-832.

6. - Some mean squares of entire functions, Proc. Amer. Math. Soc. vol. 10 (1959) pp. 833-839.

7. - Some Hilbert spaces of entire functions, Proc. Amer. Math. Soc. vol. 10 (1959) pp. 840-846.

8. - Some Hilbert spaces of entire functions, Trans. Amer. Math. Soc. vol. 96 (1960) pp. 259-295.

9. - Some Hilbert spaces of entire functions, Bull. Amer. Math. Soc. vol. 67 (1961) pp. 129-134.

10. - Some Hilbert spaces of entire functions. II, Trans. Amer. Math. Soc. vol. 99 (1961) pp. 118-152.

11. L. H. Loomis and D. V. Widder, The Poisson representation of functions which are positive and harmonic in a half-plane, Duke Math. J. vol. 9 (1942) pp. 643-645.

12. R. E. A. C. Paley and N. Wiener, Fourier transforms in the complex domain, Amer. Math. Soc. Colloquium Publications, vol. 19, 1934.

13. M. H. Stone, Linear transformations in Hilbert space and their applications to analysis, Amer. Math. Soc. Colloquium Publications, vol. 15, 1932.

14. E. C. Titchmarsh, Introduction to the theory of Fourier integrals, Oxford, Clarendon Press, 1937.

15. E. T. Whittaker and N. Watson, $A$ course of modern analysis, third edition, Cambridge, University Press, 1920.

The Institute for Advanced Study, Princeton, New Jersey 